\title{
Modeling the Interior of Black Holes Utilizing a 4-D Spatial Blackbody Radiation Model with an Exponential Distribution
}

\author{
Christopher Pilot \\ Physics Department, Gonzaga University, Spokane, WA, USA \\ Email: pilot@gonzaga.edu
}

How to cite this paper: Pilot, C. (2019) Modeling the Interior of Black Holes Utilizing a 4-D Spatial Blackbody Radiation Model with an Exponential Distribution. Journal of High Energy Physics, Gravitation and Cosmology, 5, 953-983.

https://doi.org/10.4236/jhepgc.2019.53051

Received: June 4, 2019

Accepted: July 28, 2019

Published: July 31, 2019

Copyright ( 2019 by author(s) and Scientific Research Publishing Inc. This work is licensed under the Creative Commons Attribution International License (CC BY 4.0).

http://creativecommons.org/licenses/by/4.0/

\begin{abstract}
This is a second follow up paper on a model, which treats the black hole as a 4-D spatial ball filled with blackbody radiation. For the interior radiative mass distribution, we employ a new type of truncated probability distribution function, the exponential distribution. We find that this distribution comes closest to reproducing a singularity at the center, and yet it is finite at 4 -D radius, $r=0$. This distribution will give a constant gravitational acceleration for a test particle throughout the black hole, irrespective of radius. The 4-D gravitational acceleration is given by the expression, $g^{(4)}=-0.1 c^{2} \lambda=-0.2 \lambda G M_{R} / R$, where $R$ is the radius of the black hole, $M_{R}$ is its mass, and $\lambda$ is the exponential shape parameter, which depends only on the mass, or radius, of the black hole. We calculate the gravitational force, and the entropy within the black hole interior, as well as on its surface, identified as the event horizon, which separates 3-D from 4-D space. Similar to a truncated Gaussian distribution, the gravitational force increases discontinuously, and dramatically, upon entry into the 4-D black hole from the 3-D side. It is also radius dependent within the 4-D black hole. Moreover, the total entropy is shown to be much less than the Bekenstein result, similar to the truncated Gaussian. For the gravitational force, we obtain, $F_{G, r}^{(4)}=-0.1 c^{2} \lambda M_{r}$, where $M_{r}$ is the radiative mass enclosed within a 4-D volume of radius $r$. This unusual force law indicates that the gravitational force acting upon a layer of blackbody photons at radius $r$ is strictly proportional to the enclosed radiative energy, $M_{r} c^{2}$, contained within that radius, with $0.1 \lambda$ being the constant of proportionality. For the entropy at radius, $r$, and on the surface, we obtain an expression which is order of magnitude comparable to the truncated Normal distribution. Tables are presented for three black holes, one having a mass equal to that of the sun. The other two have masses, which are ten times that of the
\end{abstract}


sun, and $10^{6}$ solar masses. The corresponding $\lambda$ parameters are found to equal, $\left(\lambda_{A}, \lambda_{B}, \lambda_{C}\right)=\left(0.039569,0.0037996,3.01229 \times 10^{-8}\right)$, respectively. We compare these results to the truncated Gaussian distribution, which were worked out in another paper.

\section{Keywords}

Black Holes, 4-D Spatial Blackbody Radiation Model, Exponential Distribution

\section{Introduction}

This is a follow up paper, where we consider another probability distribution function (pdf), the exponential distribution, and apply it to modeling the radiative mass concentration within the interior of a black hole. We assume that the black hole is a 4-D spatial ball embedded in 3-D space, and that it is filled (packed) with blackbody photons. Moreover, the radiative energy, in all its forms, internal, pressure, and heat, makes up the radiative mass within the black hole. See references [1] [2] for a detailed description of the model, which we will not reproduce here. We will only give the briefest of sketches, and then proceed to model the interior distribution of radiative mass, employing the truncated exponential function.

In paper [1], we considered the event horizon. We conjectured/hypothesized that a rip or tear in the spatial continuum occurs there, and not at the black hole center. For it is there that we transition from 3-D space to 4-D space. A four dimensional black hole filled with blackbody radiation has many advantages over a 3-D counterpart. First, we can pack an enormous amount of mass, i.e., radiative energy within a, from our perspective, relatively small 3-D volume. Second, employing such a model, the temperature decreases precipitously when we enter the black hole. This follows from a generalized version of the Stefan-Boltzmann law, which was derived for radiative transfers between different adjoining spatial dimensions. Third, the energy density in all its forms, internal, heat, and pressure reduce significantly upon entering the 4 -D space. Fourth, we have a natural barrier which prevents wholesale entry of CMB photons, and potentially other forms of radiation, such as dark energy. Fifth, there can only be zero, or net positive radiative inflow within this model, as outside net outflow is not allowed, except through evaporative processes such as Hawking radiation. Sixth, we have an inherent positive radiative surface tension, which allows for inherent black hole formation, and keeps the event horizon positively curved and stable. The Young-Laplace relations were also considered, and generalized, within a 3-D/4-D context. All these factors/advantages were shown and derived in the first work. For a detailed description we refer the reader to reference [1].

In the $2^{\text {nd }}$ paper, reference [2], we focused on what the interior of a black hole 
might consist of. We modeled the radiative mass (energy) distribution as a probability distribution function. The specific choice was a truncated Gaussian, i.e., truncated Normal distribution. It was shown that a uniform truncated distribution, in contrast, within this space cannot accommodate gravitational forces within its interior. A temperature gradient is needed for that, which a Normal distribution can satisfy. Moreover, this gradient increases as the 4-D radius decreases. Thus the internal energy density, the heat density, the radiative pressure, the entropy density, etc. all increased, as the radius decreased. For a 4-D spatial black body, the internal energy density is proportional to the fifth power of temperature, $T_{r}^{5}$, where $T_{r}$ is defined as the temperature in an infinitesimal layer at radius, $r$. The radiative pressure and radiative heat are likewise proportional to, $T_{r}^{5}$, whereas the entropy density is proportional to $T_{r}^{4}$.

The entropy density is treated as a state variable, and it has a well-defined value within a specific layer. Utilizing our truncated Gaussian distribution, we were able to derive localized, as well as other non-localized attributes/characteristics, within the black hole. The localized quantities held within a particular layer, whereas the other properties were the cumulative effects up to, and enclosing, a specific radius. Examples of localized attributes are the mass density, the radiative pressure, the radiative force, the internal energy density, the heat density, the entropy density, etc. These hold within a specific layer at radius, $r$. Examples of non-localized quantities are enclosed mass, gravitational force, enclosed entropy, etc., all defined within an enclosing volume of 4-D radius, $r$. Our results were listed in table form. We considered three black holes, which we labeled A, B and, C. Black hole $\mathrm{A}$ has a mass equal to that of the sun, whereas black holes, $B$ and $C$ had masses, 10 times, and $10^{6}$ times, the mass of the sun, respectively. In this way comparisons between various massive black holes could be made. The localized quantities were given in Tables 1-3, respectively, as a function of radius. The non-localized characteristics were tabulated under Tables 4-6, respectively for the three black holes under consideration.

Of particular interest in the second paper was the probability distribution function [3]-[9]. All results relating to the interior of the black hole depended on this choice of function. Quite simply, the pdf told us how the black hole is packed, radiative energy (mass) wise. This determined the internal forces, both radiative and gravitational, the pressures and the densities within the black hole. We chose a truncated Gaussian distribution. In this paper we will select another pdf, the truncated exponential, $\exp [\lambda ; 0, R]$. The question is how will the results change qualitatively and quantitatively with this choice? Are there specific peculiarities associated with this new choice? To this we now turn. We keep in mind that the truncated exponential distribution is particularly simple in that it is determined by one, and only one, parameter, $\lambda$, called the shape parameter. This parameter is defined over the range and has support, $r \in[0, R]$. Outside of this range, it does not exist. 
Table 1. For a Blackhole having 1 Solar Mass.

\begin{tabular}{|c|c|c|c|c|c|c|c|c|c|c|c|}
\hline$r / R$ & $\mathrm{f}_{\mathrm{r}} / \mathrm{f}_{\mathrm{R}}$ & $\rho_{\mathrm{r}}$ & $\mathbf{u}_{\mathrm{r}}$ & $\mathrm{p}_{\mathrm{r}}$ & $\mathrm{q}_{\mathrm{r}}$ & $\mathrm{T}_{\mathrm{r}}$ & $\mathbf{s}_{\mathrm{r}}$ & $\mathrm{F}_{\mathrm{r}}$ & $\mathrm{dT}_{\mathrm{r}} / \mathrm{dr}$ & $\mathrm{C}_{\mathrm{r}}=\mathrm{du}_{\mathrm{r}} / \mathrm{dT}_{\mathrm{r}}$ & $\mathrm{du}_{\mathrm{r}} / \mathrm{dr}$ \\
\hline & & $\left(\mathrm{kg} / \mathrm{m}^{4}\right)$ & (Joules $/ \mathrm{m}^{4}$ ) & $\begin{array}{c}\text { (Newtons/ } \\
\mathrm{m}^{3} \text { ) }\end{array}$ & (Joules $\left./ \mathrm{m}^{4}\right)$ & $(\mathrm{K})$ & $\begin{array}{c}\text { (Joules/ } \\
\left.\left(\mathrm{m}^{4} \mathrm{~K}\right)\right)\end{array}$ & (Newtons) & $(\mathrm{K} / \mathrm{m})$ & $\begin{array}{l}\text { (Joules/ } \\
\left.\mathrm{m}^{4} \mathrm{~K}\right)\end{array}$ & (Joules $\left./ \mathrm{m}^{5}\right)$ \\
\hline 0.000001 & $5.99 \mathrm{E}+49$ & $1.60 \mathrm{E}+34$ & $5.75 \mathrm{E}+50$ & $1.44 \mathrm{E}+50$ & $7.18 \mathrm{E}+50$ & $4.14 \mathrm{E}+12$ & $1.74 \mathrm{E}+38$ & $7.31 \mathrm{E}+43$ & $-8.40 \mathrm{E}+14$ & $6.95 \mathrm{E}+38$ & $-5.84 \mathrm{E}+53$ \\
\hline 0.03 & $5.40 \mathrm{E}+49$ & $5.34 \mathrm{E}+20$ & $1.92 \mathrm{E}+37$ & $4.80 \mathrm{E}+36$ & $2.40 \mathrm{E}+37$ & $8.34 \mathrm{E}+09$ & $2.88 \mathrm{E}+27$ & $6.59 \mathrm{E}+43$ & $-6.04 \mathrm{E}+07$ & $1.15 \mathrm{E}+28$ & $-6.95 \mathrm{E}+35$ \\
\hline 0.06 & $3.96 \mathrm{E}+49$ & $4.90 \mathrm{E}+19$ & $1.76 \mathrm{E}+36$ & $4.40 \mathrm{E}+35$ & $2.20 \mathrm{E}+36$ & $5.17 \mathrm{E}+09$ & $4.25 \mathrm{E}+26$ & $4.84 \mathrm{E}+43$ & $-2.23 \mathrm{E}+07$ & $1.70 \mathrm{E}+27$ & $-3.80 \mathrm{E}+34$ \\
\hline 0.09 & $2.37 \mathrm{E}+49$ & $8.67 \mathrm{E}+18$ & $3.12 \mathrm{E}+35$ & $7.79 \mathrm{E}+34$ & $3.89 \mathrm{E}+35$ & $3.66 \mathrm{E}+09$ & $1.06 \mathrm{E}+26$ & $2.89 \mathrm{E}+43$ & $-1.34 \mathrm{E}+07$ & $4.26 \mathrm{E}+26$ & $-5.69 \mathrm{E}+33$ \\
\hline 0.12 & $1.15 \mathrm{E}+49$ & $1.78 \mathrm{E}+18$ & $6.38 \mathrm{E}+34$ & $1.60 \mathrm{E}+34$ & $7.98 \mathrm{E}+34$ & $2.67 \mathrm{E}+09$ & $2.99 \mathrm{E}+25$ & $1.40 \mathrm{E}+43$ & $-9.47 \mathrm{E}+06$ & $1.20 \mathrm{E}+26$ & $-1.13 \mathrm{E}+33$ \\
\hline 0.15 & $4.54 \mathrm{E}+48$ & $3.59 \mathrm{E}+17$ & $1.29 \mathrm{E}+34$ & $3.23 \mathrm{E}+33$ & $1.61 \mathrm{E}+34$ & $1.94 \mathrm{E}+09$ & $8.34 \mathrm{E}+24$ & $5.55 \mathrm{E}+42$ & $-7.13 \mathrm{E}+06$ & $3.34 \mathrm{E}+25$ & $-2.38 \mathrm{E}+32$ \\
\hline 0.18 & $1.46 \mathrm{E}+48$ & $6.69 \mathrm{E}+16$ & $2.40 \mathrm{E}+33$ & $6.01 \mathrm{E}+32$ & $3.00 \mathrm{E}+33$ & $1.38 \mathrm{E}+09$ & $2.17 \mathrm{E}+24$ & $1.78 \mathrm{E}+42$ & $-5.42 \mathrm{E}+06$ & $8.69 \mathrm{E}+24$ & $-4.71 \mathrm{E}+31$ \\
\hline 0.21 & $3.82 \mathrm{E}+47$ & $1.10 \mathrm{E}+16$ & $3.96 \mathrm{E}+32$ & $9.90 \mathrm{E}+31$ & $4.95 \mathrm{E}+32$ & $9.64 \mathrm{E}+08$ & $5.13 \mathrm{E}+23$ & $4.66 \mathrm{E}+41$ & $-4.08 \mathrm{E}+06$ & $2.05 \mathrm{E}+24$ & $-8.37 \mathrm{E}+30$ \\
\hline 0.24 & $8.13 \mathrm{E}+46$ & $1.57 \mathrm{E}+15$ & $5.64 \mathrm{E}+31$ & $1.41 \mathrm{E}+31$ & $7.06 \mathrm{E}+31$ & $6.53 \mathrm{E}+08$ & $1.08 \mathrm{E}+23$ & $9.93 \mathrm{E}+40$ & $-2.99 \mathrm{E}+06$ & $4.32 \mathrm{E}+23$ & $-1.29 \mathrm{E}+30$ \\
\hline 0.27 & $1.41 \mathrm{E}+46$ & $1.91 \mathrm{E}+14$ & $6.86 \mathrm{E}+30$ & $1.72 \mathrm{E}+30$ & $8.58 \mathrm{E}+30$ & $4.29 \mathrm{E}+08$ & $2.00 \mathrm{E}+22$ & $1.72 \mathrm{E}+40$ & $-2.12 \mathrm{E}+06$ & $8.01 \mathrm{E}+22$ & $-1.70 \mathrm{E}+29$ \\
\hline 0.3 & $1.98 \mathrm{E}+45$ & $1.96 \mathrm{E}+13$ & $7.05 E+29$ & $1.76 \mathrm{E}+29$ & $8.81 \mathrm{E}+29$ & $2.72 \mathrm{E}+08$ & $3.24 \mathrm{E}+21$ & $2.42 \mathrm{E}+39$ & $-1.45 \mathrm{E}+06$ & $1.30 \mathrm{E}+22$ & $-1.88 \mathrm{E}+28$ \\
\hline 0.5 & $2.15 \mathrm{E}+37$ & $4.60 \mathrm{E}+04$ & $1.65 \mathrm{E}+21$ & $4.13 \mathrm{E}+20$ & $2.07 \mathrm{E}+21$ & $5.11 \mathrm{E}+06$ & $4.04 \mathrm{E}+14$ & $2.63 \mathrm{E}+31$ & $-4.17 \mathrm{E}+04$ & $1.62 \mathrm{E}+15$ & $-6.75 E+19$ \\
\hline 1 & $1.00 \mathrm{E}+00$ & $2.67 \mathrm{E}-34$ & $9.60 \mathrm{E}-18$ & $2.40 \mathrm{E}-18$ & $1.20 \mathrm{E}-17$ & $1.15 \mathrm{E}-01$ & $1.04 \mathrm{E}-16$ & $1.22 \mathrm{E}-06$ & $-1.81 \mathrm{E}-03$ & $4.17 \mathrm{E}-16$ & $-7.54 \mathrm{E}-19$ \\
\hline
\end{tabular}

Table 2. For a Blackhole having 10 Solar Masses.

\begin{tabular}{|c|c|c|c|c|c|c|c|c|c|c|c|}
\hline$r / R$ & $\mathrm{f}_{\mathrm{r}} / \mathrm{f}_{\mathrm{R}}$ & $\rho_{\mathrm{r}}$ & $\mathbf{u}_{\mathrm{r}}$ & $\mathrm{p}_{\mathrm{r}}$ & $\mathrm{q}_{\mathrm{r}}$ & $\mathrm{T}_{\mathrm{r}}$ & $\mathbf{s}_{\mathrm{r}}$ & $\mathrm{F}_{\mathrm{r}}$ & $\mathrm{dT}_{\mathrm{r}} / \mathrm{dr}$ & $\mathrm{C}_{\mathrm{r}}=\mathrm{du}_{\mathrm{r}} / \mathrm{dT}_{\mathrm{r}}$ & $\mathrm{du}_{\mathrm{r}} / \mathrm{dr}$ \\
\hline & & $\left(\mathrm{kg} / \mathrm{m}^{4}\right)$ & (Joules $/ \mathrm{m}^{4}$ ) & $\begin{array}{c}\text { (Newtons/ } \\
\mathrm{m}^{3} \text { ) }\end{array}$ & $\left(\right.$ Joules $\left./ \mathrm{m}^{4}\right)$ & $(\mathrm{K})$ & $\begin{array}{c}\text { (Joules/ } \\
\left.\left(\mathrm{m}^{4} \mathrm{~K}\right)\right)\end{array}$ & (Newtons) & $(\mathrm{K} / \mathrm{m})$ & $\begin{array}{c}\text { (Joules/ } \\
\left.\mathrm{m}^{4} \mathrm{~K}\right)\end{array}$ & $\left(\right.$ Joules $/ \mathrm{m}^{5}$ ) \\
\hline 0.000001 & $5.87 \mathrm{E}+47$ & $1.57 \mathrm{E}+31$ & $5.63 \mathrm{E}+47$ & $1.41 \mathrm{E}+47$ & $7.04 \mathrm{E}+47$ & $1.03 \mathrm{E}+12$ & $6.80 \mathrm{E}+35$ & $7.16 \mathrm{E}+43$ & $-2.10 \mathrm{E}+13$ & $2.72 \mathrm{E}+36$ & $-5.72 \mathrm{E}+49$ \\
\hline 0.03 & $5.31 \mathrm{E}+47$ & $5.25 \mathrm{E}+17$ & $1.89 \mathrm{E}+34$ & $4.72 \mathrm{E}+33$ & $2.36 \mathrm{E}+34$ & $2.09 \mathrm{E}+09$ & $1.13 \mathrm{E}+25$ & $6.49 \mathrm{E}+43$ & $-1.51 \mathrm{E}+06$ & $4.52 \mathrm{E}+25$ & $-6.81 E+31$ \\
\hline 0.06 & $3.95 \mathrm{E}+47$ & $4.88 \mathrm{E}+16$ & $1.75 E+33$ & $4.39 \mathrm{E}+32$ & $2.19 \mathrm{E}+33$ & $1.30 \mathrm{E}+09$ & $1.69 \mathrm{E}+24$ & $4.82 \mathrm{E}+43$ & $-5.56 \mathrm{E}+05$ & $6.75 \mathrm{E}+24$ & $-3.75 \mathrm{E}+30$ \\
\hline 0.09 & $2.41 \mathrm{E}+47$ & $8.81 \mathrm{E}+15$ & $3.17 \mathrm{E}+32$ & $7.92 \mathrm{E}+31$ & $3.96 \mathrm{E}+32$ & $9.22 \mathrm{E}+08$ & $4.29 \mathrm{E}+23$ & $2.94 \mathrm{E}+43$ & $-3.32 \mathrm{E}+05$ & $1.72 \mathrm{E}+24$ & $-5.70 \mathrm{E}+29$ \\
\hline 0.12 & $1.20 \mathrm{E}+47$ & $1.86 \mathrm{E}+15$ & $6.68 \mathrm{E}+31$ & $1.67 \mathrm{E}+31$ & $8.36 \mathrm{E}+31$ & $6.76 \mathrm{E}+08$ & $1.24 \mathrm{E}+23$ & $1.47 \mathrm{E}+43$ & $-2.35 \mathrm{E}+05$ & $4.95 \mathrm{E}+23$ & $-1.16 \mathrm{E}+29$ \\
\hline 0.15 & $4.94 \mathrm{E}+46$ & $3.91 \mathrm{E}+14$ & $1.40 \mathrm{E}+31$ & $3.51 \mathrm{E}+30$ & $1.76 \mathrm{E}+31$ & $4.95 \mathrm{E}+08$ & $3.55 \mathrm{E}+22$ & $6.03 \mathrm{E}+42$ & $-1.77 \mathrm{E}+05$ & $1.42 \mathrm{E}+23$ & $-2.52 \mathrm{E}+28$ \\
\hline 0.18 & $1.66 \mathrm{E}+46$ & $7.61 \mathrm{E}+13$ & $2.74 \mathrm{E}+30$ & $6.84 \mathrm{E}+29$ & $3.42 \mathrm{E}+30$ & $3.57 \mathrm{E}+08$ & $9.59 \mathrm{E}+21$ & $2.03 \mathrm{E}+42$ & $-1.36 \mathrm{E}+05$ & $3.84 \mathrm{E}+22$ & $-5.21 \mathrm{E}+27$ \\
\hline 0.21 & $4.59 \mathrm{E}+45$ & $1.32 \mathrm{E}+13$ & $4.76 \mathrm{E}+29$ & $1.19 \mathrm{E}+29$ & $5.94 \mathrm{E}+29$ & $2.51 \mathrm{E}+08$ & $2.37 \mathrm{E}+21$ & $5.60 \mathrm{E}+41$ & $-1.03 \mathrm{E}+05$ & $9.46 \mathrm{E}+21$ & $-9.74 \mathrm{E}+26$ \\
\hline 0.24 & $1.04 \mathrm{E}+45$ & $2.01 \mathrm{E}+12$ & $7.22 \mathrm{E}+28$ & $1.80 \mathrm{E}+28$ & $9.02 \mathrm{E}+28$ & $1.72 \mathrm{E}+08$ & $5.24 \mathrm{E}+20$ & $1.27 \mathrm{E}+41$ & $-7.62 \mathrm{E}+04$ & $2.09 \mathrm{E}+21$ & $-1.60 \mathrm{E}+26$ \\
\hline 0.27 & $1.93 \mathrm{E}+44$ & $2.62 \mathrm{E}+11$ & $9.42 \mathrm{E}+27$ & $2.36 \mathrm{E}+27$ & $1.18 \mathrm{E}+28$ & $1.15 \mathrm{E}+08$ & $1.03 \mathrm{E}+20$ & $2.36 \mathrm{E}+40$ & $-5.47 \mathrm{E}+04$ & $4.11 \mathrm{E}+20$ & $-2.25 \mathrm{E}+25$ \\
\hline 0.3 & $2.95 \mathrm{E}+43$ & $2.91 \mathrm{E}+10$ & $1.05 \mathrm{E}+27$ & $2.62 \mathrm{E}+26$ & $1.31 \mathrm{E}+27$ & $7.39 \mathrm{E}+07$ & $1.77 \mathrm{E}+19$ & $3.60 \mathrm{E}+39$ & $-3.80 \mathrm{E}+04$ & $7.08 \mathrm{E}+19$ & $-2.69 \mathrm{E}+24$ \\
\hline 0.5 & $6.70 \mathrm{E}+35$ & $1.43 \mathrm{E}+02$ & $5.15 \mathrm{E}+18$ & $1.29 \mathrm{E}+18$ & $6.43 \mathrm{E}+18$ & $1.61 \mathrm{E}+06$ & $3.99 \mathrm{E}+12$ & $8.18 \mathrm{E}+31$ & $-1.26 \mathrm{E}+03$ & $1.60 \mathrm{E}+13$ & $-2.02 \mathrm{E}+16$ \\
\hline 1 & $1.00 \mathrm{E}+00$ & $2.67 \mathrm{E}-35$ & $9.60 \mathrm{E}-19$ & $2.40 \mathrm{E}-19$ & $1.20 \mathrm{E}-18$ & $7.26 \mathrm{E}-02$ & $1.65 \mathrm{E}-17$ & $1.22 \mathrm{E}-04$ & $-1.10 \mathrm{E}-04$ & $6.61 \mathrm{E}-17$ & $-7.24 \mathrm{E}-21$ \\
\hline
\end{tabular}


Table 3. For a Blackhole having $10^{6}$ Solar Masses.

\begin{tabular}{|c|c|c|c|c|c|c|c|c|c|c|c|}
\hline $\mathrm{r} / \mathrm{R}$ & $f_{r} / f_{R}$ & $\rho_{\mathrm{r}}$ & $\mathbf{u}_{\mathrm{r}}$ & $\mathrm{p}_{\mathrm{r}}$ & $\mathrm{q}_{\mathrm{r}}$ & $\mathrm{T}_{\mathrm{r}}$ & $\mathbf{s}_{\mathrm{r}}$ & $\mathrm{F}_{\mathrm{r}}$ & $\mathrm{dT}_{\mathrm{r}} / \mathrm{dr}$ & $\mathrm{C}_{\mathrm{r}}=\mathrm{du}_{\mathrm{r}} / \mathrm{dT}_{\mathrm{r}}$ & $\mathrm{du}_{\mathrm{r}} / \mathrm{dr}$ \\
\hline & & $\left(\mathrm{kg} / \mathrm{m}^{4}\right)$ & (Joules $/ \mathrm{m}^{4}$ ) & $\begin{array}{c}\text { (Newtons/ } \\
\mathrm{m}^{3} \text { ) }\end{array}$ & (Joules $/ \mathrm{m}^{4}$ ) & $(\mathrm{K})$ & $\begin{array}{l}\text { (Joules/ } \\
\left.\left(\mathrm{m}^{4} \mathrm{~K}\right)\right)\end{array}$ & (Newtons) & $(\mathrm{K} / \mathrm{m})$ & $\begin{array}{c}\text { (Joules/ } \\
\left.\mathrm{m}^{4} \mathrm{~K}\right)\end{array}$ & $\begin{array}{c}\text { (Joules/ } \\
\left.\mathrm{m}^{5}\right)\end{array}$ \\
\hline 0.000001 & $5.21 \mathrm{E}+37$ & $1.39 \mathrm{E}+16$ & $5.00 \mathrm{E}+32$ & $1.25 \mathrm{E}+32$ & $6.25 \mathrm{E}+32$ & $1.01 \mathrm{E}+09$ & $6.19 \mathrm{E}+23$ & $6.36 \mathrm{E}+43$ & $-2.05 \mathrm{E}+05$ & $2.48 \mathrm{E}+24$ & $-5.08 \mathrm{E}+29$ \\
\hline 0.03 & $4.82 \mathrm{E}+37$ & $4.77 \mathrm{E}+02$ & $1.71 \mathrm{E}+19$ & $4.28 \mathrm{E}+18$ & $2.14 \mathrm{E}+19$ & $2.05 \mathrm{E}+06$ & $1.05 \mathrm{E}+13$ & $5.89 \mathrm{E}+43$ & $-1.46 \mathrm{E}-02$ & $4.18 \mathrm{E}+13$ & $-6.10 \mathrm{E}+11$ \\
\hline 0.06 & $3.81 \mathrm{E}+37$ & $4.71 \mathrm{E}+01$ & $1.69 \mathrm{E}+18$ & $4.24 \mathrm{E}+17$ & $2.12 \mathrm{E}+18$ & $1.29 \mathrm{E}+06$ & $1.64 \mathrm{E}+12$ & $4.65 \mathrm{E}+43$ & $-5.28 \mathrm{E}-03$ & $6.57 \mathrm{E}+12$ & $-3.47 \mathrm{E}+10$ \\
\hline 0.09 & $2.58 \mathrm{E}+37$ & $9.45 \mathrm{E}+00$ & $3.40 \mathrm{E}+17$ & $8.49 \mathrm{E}+16$ & $4.24 \mathrm{E}+17$ & $9.35 \mathrm{E}+05$ & $4.54 \mathrm{E}+11$ & $3.15 E+43$ & $-3.10 \mathrm{E}-03$ & $1.82 \mathrm{E}+12$ & $-5.63 \mathrm{E}+09$ \\
\hline 0.12 & $1.49 \mathrm{E}+37$ & $2.31 \mathrm{E}+00$ & $8.29 \mathrm{E}+16$ & $2.07 \mathrm{E}+16$ & $1.04 \mathrm{E}+17$ & $7.05 \mathrm{E}+05$ & $1.47 \mathrm{E}+11$ & $1.82 \mathrm{E}+43$ & $-2.19 \mathrm{E}-03$ & $5.88 \mathrm{E}+11$ & $-1.29 \mathrm{E}+09$ \\
\hline 0.15 & $7.39 \mathrm{E}+36$ & $5.84 \mathrm{E}-01$ & $2.10 \mathrm{E}+16$ & $5.25 \mathrm{E}+15$ & $2.63 \mathrm{E}+16$ & $5.36 \mathrm{E}+05$ & $4.90 \mathrm{E}+10$ & $9.02 \mathrm{E}+42$ & $-1.67 \mathrm{E}-03$ & $1.96 \mathrm{E}+11$ & $-3.27 \mathrm{E}+08$ \\
\hline 0.18 & $3.13 E+36$ & $1.43 \mathrm{E}-01$ & $5.14 \mathrm{E}+15$ & $1.29 \mathrm{E}+15$ & $6.43 E+15$ & $4.05 \mathrm{E}+05$ & $1.59 \mathrm{E}+10$ & $3.82 \mathrm{E}+42$ & $-1.31 \mathrm{E}-03$ & $6.36 \mathrm{E}+10$ & $-8.35 \mathrm{E}+07$ \\
\hline 0.21 & $1.13 \mathrm{E}+36$ & $3.26 \mathrm{E}-02$ & $1.17 \mathrm{E}+15$ & $2.93 \mathrm{E}+14$ & $1.47 \mathrm{E}+15$ & $3.01 \mathrm{E}+05$ & $4.87 \mathrm{E}+09$ & $1.38 \mathrm{E}+42$ & $-1.03 \mathrm{E}-03$ & $1.95 \mathrm{E}+10$ & $-2.02 \mathrm{E}+07$ \\
\hline 0.24 & $3.50 \mathrm{E}+35$ & $6.77 \mathrm{E}-03$ & $2.43 \mathrm{E}+14$ & $6.08 \mathrm{E}+13$ & $3.04 \mathrm{E}+14$ & $2.20 \mathrm{E}+05$ & $1.38 \mathrm{E}+09$ & $4.28 \mathrm{E}+41$ & $-8.06 \mathrm{E}-04$ & $5.53 \mathrm{E}+09$ & $-4.46 \mathrm{E}+06$ \\
\hline 0.27 & $9.28 \mathrm{E}+34$ & $1.26 \mathrm{E}-03$ & $4.52 \mathrm{E}+13$ & $1.13 \mathrm{E}+13$ & $5.65 \mathrm{E}+13$ & $1.57 \mathrm{E}+05$ & $3.60 \mathrm{E}+08$ & $1.13 \mathrm{E}+41$ & $-6.16 \mathrm{E}-04$ & $1.44 \mathrm{E}+09$ & $-8.88 \mathrm{E}+05$ \\
\hline 0.3 & $2.10 \mathrm{E}+34$ & $2.08 \mathrm{E}-04$ & $7.47 \mathrm{E}+12$ & $1.87 \mathrm{E}+12$ & $9.34 \mathrm{E}+12$ & $1.09 \mathrm{E}+05$ & $8.53 \mathrm{E}+07$ & $2.57 \mathrm{E}+40$ & $-4.60 \mathrm{E}-04$ & $3.41 \mathrm{E}+08$ & $-1.57 \mathrm{E}+05$ \\
\hline 0.5 & $1.94 \mathrm{E}+28$ & $4.14 \mathrm{E}-11$ & $1.49 \mathrm{E}+06$ & $3.72 \mathrm{E}+05$ & $1.86 \mathrm{E}+06$ & $5.00 \mathrm{E}+03$ & $3.72 \mathrm{E}+02$ & $2.37 \mathrm{E}+34$ & $-3.15 \mathrm{E}-05$ & $1.49 \mathrm{E}+03$ & $-4.68 \mathrm{E}-02$ \\
\hline 1 & $1.00 \mathrm{E}+00$ & $2.67 \mathrm{E}-40$ & $9.60 \mathrm{E}-24$ & $2.40 \mathrm{E}-24$ & $1.20 \mathrm{E}-23$ & $7.26 \mathrm{E}-03$ & $1.65 \mathrm{E}-21$ & $1.22 \mathrm{E}+06$ & $-8.69 \mathrm{E}-11$ & $6.61 \mathrm{E}-21$ & $-5.74 \mathrm{E}-31$ \\
\hline
\end{tabular}

Table 4. For a Blackhole having 1 Solar Mass.

\begin{tabular}{|c|c|c|c|c|c|c|c|c|c|c|c|}
\hline $\mathrm{r} / \mathrm{R}$ & $\mathbf{F}_{\mathrm{r}}^{\mathrm{CDF}}$ & Mass $=\mathrm{M}_{\mathrm{r}}$ & $\mathrm{U}_{\mathrm{r}}$ & $\mathrm{Q}_{\mathrm{r}}$ & $\mathrm{W}_{\mathrm{r}}$ & $\mathrm{G}_{\mathrm{r}} \mathrm{M}_{\mathrm{r}}$ & $\mathrm{G}_{\mathrm{r}}$ & $\mathrm{g}_{\mathrm{r}}$ & $\varphi_{\mathrm{G}, \mathrm{r}}$ & $\mathbf{F}_{\mathrm{G}, \mathrm{r}}$ & $\mathrm{S}_{\mathrm{r}}$ \\
\hline & & $(\mathrm{kg})$ & (Joules) & (Joules) & (Joules) & $\left(\mathrm{N} \mathrm{m}^{3} / \mathrm{kg}\right)$ & $\left(\mathrm{N} \mathrm{m}^{3} / \mathrm{kg}^{2}\right)$ & $\left(\mathrm{m} / \mathrm{s}^{2}\right)$ & (Joules/kg) & (Newtons) & (Joules/K) \\
\hline 0.000001 & $1.21 \mathrm{E}-05$ & $2.40 \mathrm{E}+25$ & $8.64 \mathrm{E}+41$ & $1.08 \mathrm{E}+42$ & $2.16 \mathrm{E}+41$ & $1.80 \mathrm{E}+01$ & $7.48 \mathrm{E}-25$ & $-6.97 \mathrm{E}+08$ & $-1.08 \mathrm{E}+18$ & $8.37 \mathrm{E}+33$ & $1.63 \mathrm{E}+29$ \\
\hline 0.03 & $3.50 \mathrm{E}-01$ & $6.97 \mathrm{E}+29$ & $2.50 \mathrm{E}+46$ & $3.13 \mathrm{E}+46$ & $6.26 \mathrm{E}+45$ & $1.46 \mathrm{E}+19$ & $2.09 \mathrm{E}-11$ & $-2.09 E+13$ & $-1.07 \mathrm{E}+18$ & $7.16 \mathrm{E}+42$ & $2.29 \mathrm{E}+36$ \\
\hline 0.06 & $6.36 \mathrm{E}-01$ & $1.27 \mathrm{E}+30$ & $4.55 \mathrm{E}+46$ & $5.69 \mathrm{E}+46$ & $1.14 \mathrm{E}+46$ & $2.33 \mathrm{E}+20$ & $1.84 \mathrm{E}-10$ & $-4.18 \mathrm{E}+13$ & $-1.07 \mathrm{E}+18$ & $2.47 \mathrm{E}+43$ & $6.25 \mathrm{E}+36$ \\
\hline 0.09 & $8.27 \mathrm{E}-01$ & $1.64 \mathrm{E}+30$ & $5.91 \mathrm{E}+46$ & $7.39 \mathrm{E}+46$ & $1.48 \mathrm{E}+46$ & $1.18 \mathrm{E}+21$ & $7.17 \mathrm{E}-10$ & $-6.28 \mathrm{E}+13$ & $-1.07 \mathrm{E}+18$ & $4.42 \mathrm{E}+43$ & $1.01 \mathrm{E}+37$ \\
\hline 0.12 & $9.31 \mathrm{E}-01$ & $1.85 \mathrm{E}+30$ & $6.66 \mathrm{E}+46$ & $8.32 \mathrm{E}+46$ & $1.66 \mathrm{E}+46$ & $3.73 \mathrm{E}+21$ & $2.01 \mathrm{E}-09$ & $-8.37 \mathrm{E}+13$ & $-1.06 \mathrm{E}+18$ & $5.90 \mathrm{E}+43$ & $1.31 \mathrm{E}+37$ \\
\hline 0.15 & $9.77 \mathrm{E}-01$ & $1.94 \mathrm{E}+30$ & $6.99 \mathrm{E}+46$ & $8.73 E+46$ & $1.75 \mathrm{E}+46$ & $9.10 \mathrm{E}+21$ & $4.68 \mathrm{E}-09$ & $-1.05 \mathrm{E}+14$ & $-1.05 \mathrm{E}+18$ & $6.75 \mathrm{E}+43$ & $1.48 \mathrm{E}+37$ \\
\hline 0.21 & $9.99 \mathrm{E}-01$ & $1.99 \mathrm{E}+30$ & $7.14 \mathrm{E}+46$ & $8.92 \mathrm{E}+46$ & $1.78 \mathrm{E}+46$ & $3.50 \mathrm{E}+22$ & $1.76 \mathrm{E}-08$ & $-1.46 \mathrm{E}+14$ & $-1.03 \mathrm{E}+18$ & $7.26 \mathrm{E}+43$ & $1.61 \mathrm{E}+37$ \\
\hline 0.24 & $1.00 \mathrm{E}+00$ & $1.99 \mathrm{E}+30$ & $7.15 \mathrm{E}+46$ & $8.94 \mathrm{E}+46$ & $1.79 \mathrm{E}+46$ & $5.96 \mathrm{E}+22$ & $3.00 \mathrm{E}-08$ & $-1.67 \mathrm{E}+14$ & $-1.02 \mathrm{E}+18$ & $7.30 \mathrm{E}+43$ & $1.62 \mathrm{E}+37$ \\
\hline 0.27 & $1.00 \mathrm{E}+00$ & $1.99 \mathrm{E}+30$ & $7.15 \mathrm{E}+46$ & $8.94 \mathrm{E}+46$ & $1.79 \mathrm{E}+46$ & $9.55 \mathrm{E}+22$ & $4.80 \mathrm{E}-08$ & $-1.88 \mathrm{E}+14$ & $-1.00 \mathrm{E}+18$ & $7.30 \mathrm{E}+43$ & $1.63 \mathrm{E}+37$ \\
\hline 0.3 & $1.00 \mathrm{E}+00$ & $1.99 \mathrm{E}+30$ & $7.15 \mathrm{E}+46$ & $8.94 \mathrm{E}+46$ & $1.79 \mathrm{E}+46$ & $1.46 \mathrm{E}+23$ & $7.32 \mathrm{E}-08$ & $-2.09 \mathrm{E}+14$ & $-9.82 \mathrm{E}+17$ & $7.30 \mathrm{E}+43$ & $1.63 \mathrm{E}+37$ \\
\hline 0.5 & $1.00 \mathrm{E}+00$ & $1.99 \mathrm{E}+30$ & $7.15 \mathrm{E}+46$ & $8.94 \mathrm{E}+46$ & $1.79 \mathrm{E}+46$ & $1.12 \mathrm{E}+24$ & $5.65 \mathrm{E}-07$ & $-3.49 \mathrm{E}+14$ & $-8.18 \mathrm{E}+17$ & $7.30 \mathrm{E}+43$ & $1.63 \mathrm{E}+37$ \\
\hline 1 & $1.00 \mathrm{E}+00$ & $1.99 \mathrm{E}+30$ & $7.15 E+46$ & $8.94 \mathrm{E}+46$ & $1.79 \mathrm{E}+46$ & $1.80 \mathrm{E}+25$ & $9.04 \mathrm{E}-06$ & $-6.97 \mathrm{E}+14$ & $-4.49 E+16$ & $7.30 \mathrm{E}+43$ & $1.63 \mathrm{E}+37$ \\
\hline
\end{tabular}


Table 5. For a Blackhole having 10 Solar Masses.

\begin{tabular}{|c|c|c|c|c|c|c|c|c|c|c|c|}
\hline $\mathrm{r} / \mathrm{R}$ & $\mathbf{F}_{\mathrm{r}}^{\mathrm{CDF}}$ & Mass $=\mathrm{M}_{\mathrm{r}}$ & $\mathrm{U}_{\mathrm{r}}$ & $\mathrm{Q}_{\mathrm{r}}$ & $\mathrm{W}_{\mathrm{r}}$ & $\mathrm{G}_{\mathrm{r}} \mathrm{M}_{\mathrm{r}}$ & $\mathrm{G}_{\mathrm{r}}$ & $\mathrm{g}_{\mathrm{r}}$ & $\varphi_{\mathrm{G}, \mathrm{r}}$ & $\mathbf{F}_{\mathrm{G}, \mathrm{r}}$ & $\mathrm{S}_{\mathrm{r}}$ \\
\hline & & $(\mathrm{kg})$ & (Joules) & (Joules) & (Joules) & $\left(\mathrm{N} \mathrm{m}^{3} / \mathrm{kg}\right)$ & $\left(\mathrm{N} \mathrm{m} \mathrm{m}^{3} / \mathrm{kg}^{2}\right)$ & $\left(\mathrm{m} / \mathrm{s}^{2}\right)$ & (Joules/kg) & (Newtons) & (Joules/K) \\
\hline 0.000001 & $1.18 \mathrm{E}-05$ & $2.35 E+26$ & $8.46 \mathrm{E}+42$ & $1.06 \mathrm{E}+43$ & $2.12 \mathrm{E}+42$ & $1.73 \mathrm{E}+03$ & $7.33 \mathrm{E}-24$ & $-6.69 E+07$ & $-1.03 \mathrm{E}+18$ & $7.87 \mathrm{E}+35$ & $6.38 \mathrm{E}+32$ \\
\hline 0.03 & $3.44 \mathrm{E}-01$ & $6.84 \mathrm{E}+30$ & $2.46 \mathrm{E}+47$ & $3.07 \mathrm{E}+47$ & $6.14 \mathrm{E}+46$ & $1.40 \mathrm{E}+21$ & $2.04 \mathrm{E}-10$ & $-2.01 \mathrm{E}+12$ & $-1.03 \mathrm{E}+18$ & $6.74 \mathrm{E}+44$ & $8.98 E+39$ \\
\hline 0.06 & $6.26 \mathrm{E}-01$ & $1.25 \mathrm{E}+31$ & $4.48 \mathrm{E}+47$ & $5.60 \mathrm{E}+47$ & $1.12 \mathrm{E}+47$ & $2.24 \mathrm{E}+22$ & $1.79 \mathrm{E}-09$ & $-4.02 \mathrm{E}+12$ & $-1.03 \mathrm{E}+18$ & $2.34 \mathrm{E}+45$ & $2.46 \mathrm{E}+40$ \\
\hline 0.09 & $8.18 \mathrm{E}-01$ & $1.63 \mathrm{E}+31$ & $5.85 \mathrm{E}+47$ & $7.31 \mathrm{E}+47$ & $1.46 \mathrm{E}+47$ & $1.13 \mathrm{E}+23$ & $6.96 \mathrm{E}-09$ & $-6.02 \mathrm{E}+12$ & $-1.03 \mathrm{E}+18$ & $4.22 \mathrm{E}+45$ & $4.02 \mathrm{E}+40$ \\
\hline 0.12 & $9.25 \mathrm{E}-01$ & $1.84 \mathrm{E}+31$ & $6.61 \mathrm{E}+47$ & $8.27 \mathrm{E}+47$ & $1.65 \mathrm{E}+47$ & $3.58 \mathrm{E}+23$ & $1.94 \mathrm{E}-08$ & $-8.03 E+12$ & $-1.02 \mathrm{E}+18$ & $5.69 \mathrm{E}+45$ & $5.21 \mathrm{E}+40$ \\
\hline 0.15 & $9.74 \mathrm{E}-01$ & $1.94 \mathrm{E}+31$ & $6.96 \mathrm{E}+47$ & $8.70 \mathrm{E}+47$ & $1.74 \mathrm{E}+47$ & $8.73 E+23$ & $4.51 \mathrm{E}-08$ & $-1.00 \mathrm{E}+13$ & $-1.01 \mathrm{E}+18$ & $6.55 \mathrm{E}+45$ & $5.95 \mathrm{E}+40$ \\
\hline 0.21 & $9.98 \mathrm{E}-01$ & $1.99 \mathrm{E}+31$ & $7.14 \mathrm{E}+47$ & $8.92 \mathrm{E}+47$ & $1.78 \mathrm{E}+47$ & $3.36 \mathrm{E}+24$ & $1.69 \mathrm{E}-07$ & $-1.41 \mathrm{E}+13$ & $-9.90 \mathrm{E}+17$ & $7.10 \mathrm{E}+45$ & $6.50 \mathrm{E}+40$ \\
\hline 0.24 & $1.00 \mathrm{E}+00$ & $1.99 \mathrm{E}+31$ & $7.15 E+47$ & $8.93 \mathrm{E}+47$ & $1.79 \mathrm{E}+47$ & $5.72 \mathrm{E}+24$ & $2.88 \mathrm{E}-07$ & $-1.61 \mathrm{E}+13$ & $-9.77 \mathrm{E}+17$ & $7.14 \mathrm{E}+45$ & $6.56 \mathrm{E}+40$ \\
\hline 0.27 & $1.00 \mathrm{E}+00$ & $1.99 \mathrm{E}+31$ & $7.15 \mathrm{E}+47$ & $8.94 \mathrm{E}+47$ & $1.79 \mathrm{E}+47$ & $9.17 \mathrm{E}+24$ & $4.61 \mathrm{E}-07$ & $-1.81 \mathrm{E}+13$ & $-9.61 \mathrm{E}+17$ & $7.15 \mathrm{E}+45$ & $6.58 \mathrm{E}+40$ \\
\hline 0.3 & $1.00 \mathrm{E}+00$ & $1.99 \mathrm{E}+31$ & $7.15 E+47$ & $8.94 \mathrm{E}+47$ & $1.79 \mathrm{E}+47$ & $1.40 \mathrm{E}+25$ & $7.03 \mathrm{E}-07$ & $-2.01 \mathrm{E}+13$ & $-9.45 \mathrm{E}+17$ & $7.16 \mathrm{E}+45$ & $6.59 \mathrm{E}+40$ \\
\hline 0.5 & $1.00 \mathrm{E}+00$ & $1.99 \mathrm{E}+31$ & $7.15 E+47$ & $8.94 \mathrm{E}+47$ & $1.79 \mathrm{E}+47$ & $1.08 \mathrm{E}+26$ & $5.42 \mathrm{E}-06$ & $-3.35 E+13$ & $-7.86 \mathrm{E}+17$ & $7.16 \mathrm{E}+45$ & $6.59 \mathrm{E}+40$ \\
\hline 1 & $1.00 \mathrm{E}+00$ & $1.99 \mathrm{E}+31$ & $7.15 \mathrm{E}+47$ & $8.94 \mathrm{E}+47$ & $1.79 \mathrm{E}+47$ & $1.73 \mathrm{E}+27$ & $8.67 \mathrm{E}-05$ & $-6.69 E+13$ & $-4.49 \mathrm{E}+16$ & $7.16 \mathrm{E}+45$ & $6.59 \mathrm{E}+40$ \\
\hline
\end{tabular}

Table 6. For a Blackhole having $10^{6}$ Solar Masses.

\begin{tabular}{|c|c|c|c|c|c|c|c|c|c|c|c|}
\hline $\mathrm{r} / \mathrm{R}$ & $\mathbf{F}_{\mathrm{r}}^{\mathrm{CDF}}$ & Mass $=\mathbf{M}_{\mathrm{r}}$ & $\mathrm{U}_{\mathrm{r}}$ & $\mathrm{Q}_{\mathrm{r}}$ & $\mathrm{W}_{\mathrm{r}}$ & $\mathrm{G}_{\mathrm{r}} \mathrm{M}_{\mathrm{r}}$ & $\mathrm{G}_{\mathrm{r}}$ & $\mathrm{g}_{\mathrm{r}}$ & $\varphi_{\mathrm{G}, \mathrm{r}}$ & $\mathbf{F}_{\mathrm{G}, \mathrm{r}}$ & $\mathrm{S}_{\mathrm{r}}$ \\
\hline & & (kg) & (Joules) & (Joules) & (Joules) & $\left(\mathrm{N} \mathrm{m}^{3} / \mathrm{kg}\right)$ & $\left(\mathrm{N} \mathrm{m}^{3} / \mathrm{kg}^{2}\right)$ & $\left(\mathrm{m} / \mathrm{s}^{2}\right)$ & (Joules/kg) & (Newtons) & (Joules/K) \\
\hline 0.000001 & $1.05 \mathrm{E}-05$ & $2.09 \mathrm{E}+31$ & $7.52 \mathrm{E}+47$ & $9.40 \mathrm{E}+47$ & $1.88 \mathrm{E}+47$ & $1.36 \mathrm{E}+13$ & $6.51 \mathrm{E}-19$ & $-5.28 \mathrm{E}+02$ & $-8.25 \mathrm{E}+17$ & $5.52 \mathrm{E}+33$ & $5.81 \mathrm{E}+38$ \\
\hline 0.03 & $3.07 \mathrm{E}-01$ & $6.11 \mathrm{E}+35$ & $2.20 \mathrm{E}+52$ & $2.75 E+52$ & $5.50 \mathrm{E}+51$ & $1.10 \mathrm{E}+31$ & $1.80 \mathrm{E}-05$ & $-1.59 \mathrm{E}+07$ & $-8.25 \mathrm{E}+17$ & $4.78 \mathrm{E}+42$ & $8.23 \mathrm{E}+45$ \\
\hline 0.06 & $5.71 \mathrm{E}-01$ & $1.14 \mathrm{E}+36$ & $4.08 \mathrm{E}+52$ & $5.10 \mathrm{E}+52$ & $1.02 \mathrm{E}+52$ & $1.77 \mathrm{E}+32$ & $1.55 \mathrm{E}-04$ & $-3.17 \mathrm{E}+07$ & $-8.23 \mathrm{E}+17$ & $1.71 \mathrm{E}+43$ & $2.30 \mathrm{E}+46$ \\
\hline 0.09 & $7.64 \mathrm{E}-01$ & $1.52 \mathrm{E}+36$ & $5.47 \mathrm{E}+52$ & $6.83 \mathrm{E}+52$ & $1.37 \mathrm{E}+52$ & $8.94 \mathrm{E}+32$ & $5.88 \mathrm{E}-04$ & $-4.76 \mathrm{E}+07$ & $-8.19 \mathrm{E}+17$ & $3.21 \mathrm{E}+43$ & $3.87 \mathrm{E}+46$ \\
\hline 0.12 & $8.86 \mathrm{E}-01$ & $1.76 \mathrm{E}+36$ & $6.34 \mathrm{E}+52$ & $7.92 \mathrm{E}+52$ & $1.58 \mathrm{E}+52$ & $2.82 \mathrm{E}+33$ & $1.60 \mathrm{E}-03$ & $-6.34 \mathrm{E}+07$ & $-8.14 \mathrm{E}+17$ & $4.54 \mathrm{E}+43$ & $5.20 \mathrm{E}+46$ \\
\hline 0.15 & $9.52 \mathrm{E}-01$ & $1.89 \mathrm{E}+36$ & $6.81 \mathrm{E}+52$ & $8.51 \mathrm{E}+52$ & $1.70 \mathrm{E}+52$ & $6.90 \mathrm{E}+33$ & $3.64 \mathrm{E}-03$ & $-7.93 \mathrm{E}+07$ & $-8.08 \mathrm{E}+17$ & $5.46 \mathrm{E}+43$ & $6.15 E+46$ \\
\hline 0.21 & $9.94 \mathrm{E}-01$ & $1.98 \mathrm{E}+36$ & $7.11 \mathrm{E}+52$ & $8.89 \mathrm{E}+52$ & $1.78 \mathrm{E}+52$ & $2.65 \mathrm{E}+34$ & $1.34 \mathrm{E}-02$ & $-1.11 \mathrm{E}+08$ & $-7.91 \mathrm{E}+17$ & $6.22 \mathrm{E}+43$ & $7.02 \mathrm{E}+46$ \\
\hline 0.24 & $9.98 \mathrm{E}-01$ & $1.99 \mathrm{E}+36$ & $7.14 \mathrm{E}+52$ & $8.92 \mathrm{E}+52$ & $1.78 \mathrm{E}+52$ & $4.52 \mathrm{E}+34$ & $2.28 \mathrm{E}-02$ & $-1.27 \mathrm{E}+08$ & $-7.81 \mathrm{E}+17$ & $6.32 \mathrm{E}+43$ & $7.16 \mathrm{E}+46$ \\
\hline 0.27 & $1.00 \mathrm{E}+00$ & $1.99 \mathrm{E}+36$ & $7.15 \mathrm{E}+52$ & $8.93 \mathrm{E}+52$ & $1.79 \mathrm{E}+52$ & $7.24 \mathrm{E}+34$ & $3.64 \mathrm{E}-02$ & $-1.43 \mathrm{E}+08$ & $-7.69 \mathrm{E}+17$ & $6.35 E+43$ & $7.21 \mathrm{E}+46$ \\
\hline 0.3 & $1.00 \mathrm{E}+00$ & $1.99 \mathrm{E}+36$ & $7.15 \mathrm{E}+52$ & $8.94 \mathrm{E}+52$ & $1.79 \mathrm{E}+52$ & $1.10 \mathrm{E}+35$ & $5.55 \mathrm{E}-02$ & $-1.59 \mathrm{E}+08$ & $-7.55 \mathrm{E}+17$ & $6.36 \mathrm{E}+43$ & $7.23 \mathrm{E}+46$ \\
\hline 0.5 & $1.00 \mathrm{E}+00$ & $1.99 \mathrm{E}+36$ & $7.15 E+52$ & $8.94 \mathrm{E}+52$ & $1.79 \mathrm{E}+52$ & $8.51 \mathrm{E}+35$ & $4.28 \mathrm{E}-01$ & $-2.64 \mathrm{E}+08$ & $-6.30 \mathrm{E}+17$ & $6.36 \mathrm{E}+43$ & $7.24 \mathrm{E}+46$ \\
\hline 1 & $1.00 \mathrm{E}+00$ & $1.99 \mathrm{E}+36$ & $7.15 E+52$ & $8.94 \mathrm{E}+52$ & $1.79 \mathrm{E}+52$ & $1.36 \mathrm{E}+37$ & $6.85 \mathrm{E}+00$ & $-5.28 \mathrm{E}+08$ & $-4.49 \mathrm{E}+16$ & $6.36 \mathrm{E}+43$ & $7.24 \mathrm{E}+46$ \\
\hline
\end{tabular}


Before we start, we point out some notable differences between our model and the conventional picture. A good survey article on the thermodynamics of black holes is given in reference [10]. The deviations between our model and the standard picture will be given in bullet form, and the list is not all-inclusive. However, these are among the most important differences, which will be seen as we move through the text. It is best to highlight them now such that misconceptions will be avoided. We note that in our model:

1) Our entropy calculation is an extensive quantity depending on 4-D spatial volume. The conventional Beckenstein-Hawking entropy depends on surface area, according to a holographic principle. For a comparable mass black hole, our extensive 4-D entropy calculation will give much reduced values for total entropy. To find the total entropy, we integrate over a 4 -D spatial volume. The Bekenstein entropy, by contrast, is proportional to the surface area of the event horizon, and therefore, an intensive variable. More correctly, it is proportional to the sum of Planck surface units enclosing the black hole.

2) There is no information loss (only information gain) upon transitioning from 3-D space (the surroundings) to 4-D space, the black hole, if only blackbody radiation is considered. Our black hole can be thought of as a special, exotic type of 4-D spatial capacitor, where pure states, in the form of blackbody radiation obeying Bose-Einstein statistics, are possible within specific layers. Not only is radiative mass stored within the 4-D black hole, but in addition, entropy and many other attributes. Entropy will increase upon massive and massless inflow. Of all isothermal radiating bodies, a black body is that unique body which produces the largest amount of entropy for a given energy. See, for example, reference [11].

3) Reduced temperatures just inside the event horizon of a black hole is a direct consequence of a spatial transition from 3-D space to 4-D space, as was shown explicitly in reference [1]. Upon transitioning from 3-D to 4-D space, surface area increases dramatically, and as a consequence, temperature decreases discontinuously, and dramatically, according to a generalized version of the Stefan-Boltzmann law. The temperature within the black hole in our model is not due to evaporative processes, such as in Hawking radiation.

4) Because the temperature changes abruptly, all blackbody attributes which depend exclusively on temperature, such as internal energy density, radiative pressures, entropy density, etc. also change abruptly at the 3-D/4-D interface, identified as the event horizon. The temperature, however, will increase as one penetrates to smaller radii within the 4 -D black hole. Thus, all these quantities which can be defined within specific layers will also increase. Ultimately, this will make up the mass of the black hole within this very simplified model.

5) Layer by layer, within the black hole, outward expansion due to radiative pressures and forces are held in check by gravitational forces pulling in. Hydrostatic equations apply as was shown explicitly in reference [2]. At the surface, we also have gravitational forces, and radiative pressure. Black holes are in thermal 
equilibrium with the surrounding $\mathrm{CMB}$ temperature when there is no net inflow. Radiative forces are such that there will always be a net positive curvature, both within, and on the surface of the black hole. This is not guaranteed on the surface for a 3-D/3-D spatial transition.

6) The gravitational force increases dramatically and abruptly as one enters the spatial 4-D black hole. This is a consequence of the spatial dimension changing. The probability distribution function chosen will determine how the radiative mass is packed or stored within the 4-D space. Typically, in 4-D space, the gravitational constant is a function of radius.

7) Since our 4-D black holes consist of blackbody radiation in this very simplified model, radiative pressure, radiative force, energy density, and entropy density are fixed very specifically in terms of temperature, $T=T(r)=T_{r}$. Derived quantities such as gravitational force, entropy, specific heat, are also fixed. Our heat capacity is not negative as in the standard picture, and it does decrease with increasing 4-D radius. Associated MKS units for heat capacity in 4-D space are Joules $/\left(\mathrm{m}^{4} \mathrm{~K}\right)$. The gravitational force in Newtons increases with increasing radius, $r \in[0, R]$.

8) By and large, we ignore Hawking radiation and other evaporative processes. This has to be worked out in another series of papers. Our temperatures just inside the event horizon depend on the size, or mass, of the black hole. They typically are much higher than the corresponding 3-D Hawking temperature, although much lower than the surrounding CMB temperature. The temperature is not constant throughout the black hole in our model, nor is it due to evaporative processes.

Clearly, these assumptions go contrary to much of what is believed to hold true for black holes. Our premise is an unconventional one, as we are dealing with a 4-D spatial object.

The general outline for this paper follows that of reference [2], although in less detail. In Section 2, we introduce the exponential pdf, and determine the shape parameters needed for each of the three black holes under consideration. We analyze the same three black holes as was done in reference [2], black holes $\mathrm{A}, \mathrm{B}$, and, $\mathrm{C}$, such that direct comparisons can be made. In this section, we use the temperature just inside the event horizon, within the 4-D black hole to find the associated shape parameters, $\left(\lambda_{A}, \lambda_{B}, \lambda_{C}\right)$. With these values, we will be in a position to map out the interior. Since we can find the various densities, the radiative pressures, and the radiative forces at the surface just inside the event horizon, we can determine these values within the interior for any arbitrary radius knowing the various shape parameters. In Section 3, we consider specifically the localized variables which hold within a particular layer, within the interior of the 4-D black hole. We are considering the internal energy density, the radiative pressure, the entropy density, the radiative force, the temperature, and the heat capacity, among other variables. The localized properties are tabulated under Tables 1-3. 
In Section 4, we derive and calculate certain cumulative properties. These are cumulative quantities, which hold within an enclosing radius. In this section, we focus on radiative mass, the gravitational force, the gravitational acceleration, and the entropy, all defined within an enclosing radius. The cumulative characteristics are listed under Tables 4-6. The key formulae are given in the text. Finally in Section 5, we discuss our results, and remark upon the similarities, as well as deviations, from the corresponding Normal distribution counterparts. We shall see that particular pdf's lead to particular ramifications.

\section{The Exponential Distribution}

We first derive a general relation which all pdf's have to obey within our model [1]. On the inside surface of a black hole, just inside the event horizon, the radiative force is given by

$$
\begin{aligned}
F_{R}^{(4)} & \equiv p_{1}^{(4)} A_{R}^{(4)}=0.8 p_{2}^{(3)} A_{R}^{(3)} \\
& =0.8 u_{2}^{(3)} / 3 A_{R}^{(3)} \\
& =0.8 a^{(3)} / 3(2.725)^{4} 4 \pi R^{2}
\end{aligned}
$$

We have assumed that the outside temperature is the CMB temperature and that, consequently, $\mathrm{d} Q / \mathrm{d} t=0$, where $\mathrm{d} Q / \mathrm{d} t$ is the radiative heat entering the black hole. An isolated, static black hole is in thermal equilibrium with its surroundings at this external temperature. In Equation, (2-1), $p_{1}^{(4)}$ is the radiative pressure just inside the event horizon, where the temperature is $T_{1}$. The black body radiative pressure just outside the event horizon, on the $3-\mathrm{D}$ side, is given by, $p_{2}^{(3)}$, and this is defined at a different temperature, $T_{2}$. For an isolated, static black hole, where, $\mathrm{d} Q / \mathrm{d} t=0$, the outside temperature, $T_{2}=2.725 \mathrm{~K}$. The quantity, $A_{R}^{(4)}=2 \pi^{2} R^{3}$, is the $4-\mathrm{D}$ surface area. The three dimensional surface area is, $A_{R}^{(3)}=4 \pi R^{2}$. The internal energy density, $u_{2}^{(3)}$, on the $3-\mathrm{D}$ side is equal to, $u_{2}^{(3)}=a^{(3)} T_{2}^{4}=4 / c \sigma^{(3)} T_{2}^{4}=4 / c \sigma^{(3)} 2.725^{4}$, where, $\sigma^{(3)}=5.68 \times 10^{-8}$ Watts $/ \mathrm{m}^{2} \cdot \mathrm{K}^{4}$, is the Stefan Boltzmann constant. The constant, $a^{(3)} \equiv 4 \sigma^{(3)} / c=7.5657 \times 10^{-16}$, in MKS units. All superscripts within brackets over a quantity refer to the spatial dimension over which the quantity is defined. If it is obvious that we are in 4 spatial dimensions, we will dispense with the superscript, such as in Sections 3, 4, and 5.

We next recognize that, according to our model, the radiative pressure just inside the 4-D black hole surface, is related to the radiative mass density, $\rho_{1}^{(4)}$, at that radius, by means of

$$
\rho_{1}^{(4)}=e_{1}^{(4)} / c^{2}=10 p_{1}^{(4)} / c^{2}
$$

In this relation, the radiative energy density, $e_{1}^{(4)}$, is defined at temperature, $T_{1}$, which is the temperature just inside the event horizon. The total 4-D energy density, which consists of internal energy density, radiative pressure, and heat density, is assumed to contribute to the radiative mass. When multiplied by a 4-D volume and/or 4-D surface area, $A_{r}^{(4)}$ the units match. For example, 


$$
e_{r}^{(4)} A_{r}^{(4)}=u_{r}^{(4)} A_{r}^{(4)}+p_{r}^{(4)} A_{r}^{(4)}+q_{r}^{(4)} A_{r}^{(4)}
$$

The heat density at radius, $r$, is designated by, $q_{r}^{(4)}$. As shown in reference [1], this can be rewritten a variety of ways,

$$
e_{r}^{(4)} A_{r}^{(4)}=10 / 4 u_{r}^{(4)} A_{r}^{(4)}=10 p_{r}^{(4)} A_{r}^{(4)}=2 q_{r}^{(4)} A_{r}^{(4)}
$$

This is due to the particular coefficients, which hold only in 4-D space,

$$
p_{r}^{(4)}=u_{r}^{(4)} / 4, q_{r}^{(4)}=5 / 4 u_{r}^{(4)}, s_{r}^{(4)}=5 / 4 u_{r}^{(4)} / T_{r}
$$

In another spatial dimension, these factors would assume different values. Equation, (2-5), gives us the relative contributions towards total energy density within any layer at radius $r$, or at a particular radius $r$. Those contributions are, $q_{r}=(1 / 2) e_{r}, \quad p_{r}=(1 / 10) e_{r}$, and, $u_{r}=(4 / 10) e_{r}$.

We multiply Equation, (2-2), by the 4 -D surface area, $A_{r}^{(4)}$. And then, we use Equation, (2-1), to eliminate, $p_{1}^{(4)} A_{R}^{(4)}$, on the right hand side of the new expression obtained. The result is

$$
\rho_{1}^{(4)} A_{R}^{(4)}=\left(10 / c^{2}\right)(8 / 3) a^{(3)}(2.725)^{4} 4 \pi R^{2}
$$

According to our model, this is precisely equal to,

$$
\rho_{1}^{(4)} A_{R}^{(4)}=f_{R} M_{R}
$$

where, $f_{R}$, is the value of the probability distribution function at $r=R$. The $R$ is the full radius of the black hole, related to its mass via the Schwarzschild relation, $R=2 G M_{R} / c^{2}$. A quick proof follows.

We introduce, quite generally, a pdf, $f_{r}$, which satisfies,

$$
\text { Probl. }\left(r_{1} \leq r \leq r_{2}\right)=\int_{l_{1}}^{r_{2}} f_{r} \mathrm{~d} r
$$

For a truncated pdf with limits or bounds, $[a, b]=[0, R]$, we thus have

$$
\text { Probl. }(0 \leq r \leq R)=\int_{0}^{R} f_{r} \mathrm{~d} r=1
$$

i.e., $100 \%$ probable. The cumulative distribution function (cdf), $F_{r}^{C D F}$, is defined as,

$$
F_{r}^{C D F} \equiv \int_{0}^{r} f_{r} \mathrm{~d} r
$$

The cdf is the sum of all probabilities starting from $r=0$ up to, and including, radius, $r$. The radiative mass within the black hole is assumed to obey such a function. In other words,

$$
M_{r}=M_{R} \int_{0}^{r} f_{r} \mathrm{~d} r=M_{R} F_{r}^{C D F}
$$

Moreover, since $M_{r}=\rho_{r} \mathrm{~d} V_{r}=\rho_{r} A_{r} \mathrm{~d} r$ is the radiative mass contained within volume, $\mathrm{d} V_{r}^{(4)}=A_{r}^{(4)} \mathrm{d} r$, within a layer between $r$ and $r+\mathrm{d} r$, we see that,

$$
M_{r}=\int_{0}^{r} \rho_{r} A_{r} \mathrm{~d} r
$$

Comparing the last two equations, we see that

$$
\rho_{r} A_{r}=M_{R} f_{r}
$$


Equation, (2-7), is a special case of relation, (2-13).

We next set the right hand side of Equation, (2-7), equal to the right hand side of Equation, (2-6), and solve for $f_{R}$. This gives

$$
f_{R}=(32 \pi / 3)\left(R^{2} / M_{R} c^{2}\right) a^{(3)}(2.725)^{4}
$$

We now make use of the Schwarzschild relation to further simplify this expression. Using the relation, $R=2 G M_{R} / c^{2}$, we eliminate $M_{R}$ in favor of $R$, to obtain for (2-14),

$$
f_{R}=\left(64 \pi G / 3 c^{2}\right) a^{(3)}(2.725)^{4} R=2.31 \times 10^{-56} R
$$

To obtain the last line, we have used the numerical value, $a^{(3)}=7.5657 \times 10^{-16}$, in MKS units. Equation, (2-15), tells us that the value of the probability distribution function is very small just inside the event horizon, on the 4-D side. Therefore, the radiative energy density, the internal energy density, the radiative pressure, etc. ... are also close to being zero. See Equation, (2-7). This holds even for very massive black holes, where the radius is significant. In fact, $f_{R}$ scales as, or is proportional to, $R$. In previous cosmological epochs, the CMB temperature was higher, and the factor sitting out in front of $R$ in Equation, (2-15), would be different. However the proportionality with respect to $R$ would not change. Even though the numerical value of $f_{R}$ is small, it is not insignificant. This value will set the boundary condition for the radiative mass profile, and internal energy profile, the heat profile, the entropy density profile, etc. within the black hole. All that has to be chosen is an appropriate pdf. Equation, (2-15), will hold for any pdf chosen.

In this paper, we consider three specific black holes. Black hole $\mathrm{A}$ has a mass equal to that of the sun. Black holes $B$ and $C$ will have masses 10 times and $10^{6}$ times the mass of the sun, respectively. For the masses chosen, the radii are given by the Schwarzschild relation, and we obtain,

$$
\begin{aligned}
& \left(M_{A}, M_{B}, M_{C}\right)=\left(1.989 \times 10^{30}, 1.989 \times 10^{31}, 1.989 \times 10^{36}\right) \mathrm{kg} \\
& \rightarrow\left(R_{A}, R_{B}, R_{C}\right)=\left(2.954 \times 10^{3}, 2.954 \times 10^{4}, 2.954 \times 10^{9}\right) \text { meters }
\end{aligned}
$$

Substituting these radii into Equation, (2-15), gives

$$
\left(f_{R, A}, f_{R, B}, f_{R, C}\right)=\left(6.8292 \times 10^{-53}, 6.8292 \times 10^{-52}, 6.8292 \times 10^{-47}\right) \text { meters }^{-1}
$$

We see very clearly that these values are quite low, even for very massive black holes.

We now specify the exponential distribution. The probability distribution function can be written in the form,

$$
f_{r}=\lambda \mathrm{e}^{-\lambda r} /\left(1-\mathrm{e}^{-\lambda R}\right)
$$

where, $\lambda$, is the shape parameter, the only parameter associated with the exponential distribution. Equation, (2-18), satisfies Equations, (2-8) and (2-9). The associated cdf defined by Equation, (2-10), is easily derived. The resulting expression is, 


$$
F_{r}^{C D F}=\left(1-\mathrm{e}^{-\lambda r}\right) /\left(1-\mathrm{e}^{-\lambda R}\right)
$$

It is obvious that, $F_{R}^{C D F}=1$. From Equation, (2-18), it follows that

$$
f_{r} / f_{R}=\mathrm{e}^{\lambda(R-r)}(\mathrm{TE}=\text { truncated exponential })
$$

This is to be contrasted to what we had previously, in reference [2], where we considered the truncated Gaussian. There the corresponding expression was,

$$
f_{r} / f_{R}=\mathrm{e}^{\left(R^{2}-r^{2}\right) / 2 \sigma^{2}}(\mathrm{TG}=\text { truncated gaussian })
$$

For the Gaussian or Normal distribution, the parameters, $(\mu, \sigma)=(0, \sigma)$, such that the mode was found at $r=0$. The quantity, $\sigma$, is the shape parameter for the TG distribution. These two expressions, Equations, (2-20) and, (2-21), will lead to very different results for what happens within the black hole, as we shall soon see.

We now will fix the exponential shape parameters for the three black holes being analyzed. We do this by specializing Equation, (2-12), to $r=R$, which places us 4-dimensionally, just inside the event horizon. There,

$$
f_{R}=\lambda \mathrm{e}^{-\lambda R} /\left(1-\mathrm{e}^{-\lambda R}\right)
$$

For our three black holes, we have specific $f_{R}$ values, as seen from Equations, (2-17). We substitute these on the left hand side of Equation, (2-22). We also know the respective radii, as these are given by our relations, (2-16). If we substitute the appropriate radii on the right hand side, then we can then use a trial and error approach to find the respective $\lambda$ values for each of the black holes. The equalities will hold in each instance only for a unique value of $\lambda$. We find that Equation, (2-22), is satisfied, if we make the following choices:

$$
\left(\lambda_{A}, \lambda_{B}, \lambda_{C}\right)=\left(0.039569,0.0037996,3.01229 \times 10^{-8}\right) \text { meters }^{-1}
$$

An analytical expression/solution for $\lambda$ in Equation, (2-22), does not seem possible as $\lambda$ occurs at various places on the right hand side.

\section{Radiative Pressure, Internal Energy Density, Heat Density, and Other Quantities within Specific Layers}

Next we construct tables for black holes A,B and C, where we calculate important attributes as a function of radii. This will give us a snapshot of how the black hole is structured internally. According to our exponential distribution, we will have a very specific profile for all the key attributes within the black hole. We consider in this section, the radiative mass density, $\rho_{r}^{(4)}$, the internal energy density, $u_{r}^{(4)}$, the heat density, $q_{r}^{(4)}$, the entropy density, $s_{r}^{(4)}$, the temperature, $T_{r}$, the radiative force, $F_{r}^{(4)}$, among other values. These are all defined in $4-\mathrm{D}$ space, and henceforth, we will suppress the superscripts unless comparisons with three dimensional quantities are made. Tables 1-3 will hold for black holes A, B and $\mathrm{C}$, respectively. We go through the same steps and follow the same format, as was done in reference [2], for a truncated Gaussian distribution. We extend the analysis to the exponential distribution. 
As was shown in reference [2], the temperature just inside the black hole is determined by the black hole mass, or equivalently, by its radius, because of the Schwarzschild proportionality. The result was,

$$
T_{1}=0.569 R^{-1 / 5}
$$

where, $T_{1}$, is the temperature just inside the black hole event horizon, at radius, $R$. Using this expression, the temperatures for the three black holes under consideration are,

$$
\left(T_{1, A}, T_{1, B}, T_{1, C}\right)=(0.115 \mathrm{~K}, 0.0726 \mathrm{~K}, 0.00726 \mathrm{~K})
$$

This allows us to find the internal energy density, the radiative pressure, the heat density, and the entropy density just inside the black hole event horizon. As shown in reference [2],

$$
\begin{gathered}
\left(u_{1, A}, u_{1, B}, u_{1, C}\right)=\left(9.60 \times 10^{-18}, 9.60 \times 10^{-19}, 9.60 \times 10^{-24}\right) \mathrm{J} / \mathrm{m}^{4} \\
\left(p_{1, A}, p_{1, B}, p_{1, C}\right)=\left(2.40 \times 10^{-18}, 2.40 \times 10^{-19}, 2.40 \times 10^{-24}\right) \mathrm{N} / \mathrm{m}^{3} \\
\left(q_{1, A}, q_{1, B}, q_{1, C}\right)=\left(1.20 \times 10^{-17}, 1.20 \times 10^{-18}, 1.20 \times 10^{-23}\right) \mathrm{J} / \mathrm{m}^{4} \\
\left(s_{1, A}, s_{1, B}, s_{1, C}\right)=\left(1.04 \times 10^{-16}, 1.65 \times 10^{-17}, 1.65 \times 10^{-21}\right) \mathrm{J} /\left(\mathrm{m}^{4} \cdot \mathrm{K}\right)
\end{gathered}
$$

These values are very small, but then the temperature, just within the event horizon is very small. According to Equation, (2-2), we can also write,

$$
\left(\rho_{1, A}, \rho_{1, B}, \rho_{1, C}\right)=\left(2.67 \times 10^{-34}, 2.67 \times 10^{-35}, 2.67 \times 10^{-40}\right) \mathrm{kg} / \mathrm{m}^{4}
$$

These are the radiative mass densities just inside the event horizon, defined in 4-D space. We note that the units in all the above quantities conform to 4-D space. The subscript " 1 " in all these quantities refers to the temperature, $T_{1}$, which is the temperature just inside the event horizon, within the black hole. This subscript could just as well be replaced by " $R$ ", as this temperature holds at radius, $R$. In other words, $u_{1, A}=u_{R, A}, u_{1, B}=u_{R, B}$, etc.. The subscripts, A, B, C, refer to black holes, $\mathrm{A}, \mathrm{B}$, and, $\mathrm{C}$, respectively.

We next make use of our relations, (2-13), and, (2-20). Combining both expressions, we see that,

$$
\left(\rho_{r} A_{r}\right) /\left(\rho_{R} A_{R}\right)=f_{r} / f_{R}=\exp [\lambda(R-r)]
$$

This equation allows us to find $\rho_{r} A_{r}$ at any radius, $r$, within the black hole. We know the radii as these are specified under Equations, (2-16). We also have specific values for the shape parameters as these are worked out in Equations, (2-23). Finally we have the mass densities at the surface. See relations, (3-7). The 4-D surface area, $A_{R}=2 \pi^{2} R^{3}$. So we have all that is needed to calculate, $\rho_{r} A_{r}$, and, $\rho_{r}$, within the interior. At any radius, $r$, the surface area, $A_{r}=2 \pi^{2} r^{3}$. Because the radius varies, we have to tabulate the above quantities in table form, and these values are listed as entries in Tables 1-3. Under column one, we consider various radii. In column two, we calculate the function indicated by Equation, (3-8). And under column three, various radiative mass density values are 
worked out.

Once $\rho_{r}$ is specified, we can easily evaluate the internal energy density, $u_{r}$, the radiative pressure, $p_{r}$, the heat density, $q_{r}$, and the temperature, $T_{r}$. These are given under columns 4, 5, 6, and, 7, respectively in Tables 1-3. We have made use of the following relations, which are easily proved once we realize that, in 4-D space [1],

$$
u_{r}^{(4)}=u^{(4)}(r)=a^{(4)} T_{r}^{5}=3 \pi / 2 c \sigma^{(4)} T_{r}^{5}=4.7481 \times 10^{-13} T_{r}^{5}
$$

The Stefan-Boltzmann constant in 4-D space [12] [13] [14] is given by, $\sigma^{(4)}=3.021 \times 10^{-5}$ Watts $/\left(\mathrm{m}^{3} \cdot \mathrm{K}^{5}\right)$. Using Equation, (3-9), and the relations, (2-2), with (2-5), we can show that,

$$
u_{r} / u_{R}=q_{r} / q_{R}=p_{r} / p_{R}=e_{r} / e_{R}=\rho_{r} / \rho_{R}=\left(T_{r} / T_{R}\right)^{5}
$$

We know $\rho_{r} / \rho_{R}$, from Equation, (3-8). The $\rho_{R}$ values are listed under Equations, (3-7). And the $\rho_{r}$ values are listed under column 3 in each of the tables. We also know the $u_{R}$ values, the $p_{R}$ values, the $q_{R}$ values, and, the $T_{R}$ values. These are given by Equations, (3-3), (3-4), (3-5), and, (3-2), respectively. Therefore, using Equations, (3-10), and, (3-8), it is possible to find, $u_{r}$, $p_{r}, q_{r}$, and, $T_{r}$, as a function of radius.

When comparing these values with those in reference [2], which holds for a truncated Gaussian, we notice a difference. The black holes are the same, and we had the same temperatures, densities, and radiative pressures at the surface. However the distribution is now different as we are using a truncated exponential, and therefore, the radiative mass density, the energy densities, and the pressures are "packed" differently within the black hole. Hence, we have the differences in the tabulated entries. We will come back to this point when we make formal comparisons between the two distributions (pdf's) in Section 5.

The entropy density, $s_{r}$, will be considered next. This is most easily evaluated by using the relation, $s_{r}=q_{r} / T_{r}$, which is a consequence of Equations, (2-5b), and, (2-5c). The $q_{r}$ and the $T_{r}$ values are listed under columns 6 , and 7 , respectively. So all we have to do is take the value in one column and divide out by its corresponding value in the other column. The $s_{r}$ values are entered under column 8 in each of the tables.

An alternative method is to recognize that the ratio

$$
\begin{aligned}
s_{r} / s_{R} & =\left(q_{r} / q_{R}\right)\left(T_{R} / T_{r}\right)=(R / r)^{3}\left(f_{r} / f_{R}\right)\left(T_{R} / T_{r}\right) \\
& =(R / r)^{3}\left(f_{r} / f_{R}\right)(R / r)^{-3 / 5}\left(f_{r} / f_{R}\right)^{-1 / 5} \\
& =(R / r)^{12 / 5}\left(f_{r} / f_{R}\right)^{4 / 5} \\
& =(R / r)^{12 / 5} \exp [0.8 \lambda(R-r)]
\end{aligned}
$$

For the first line, we used Equations (2-5b,c), (3-10), and the first equality in (3-8). For the second line, another equality in Equation, (3-10), and the first equality in (3-8), were employed. And for the $4^{\text {th }}$ line, the second equality in (3-8) was utilized. 
An even simpler formulation is to use another version of Equation, (3-10), and realize that it is also possible to write,

$$
s_{r} / s_{R}=\left(q_{r} / q_{R}\right)\left(T_{R} / T_{r}\right)=\left(T_{R} / T_{r}\right)^{4}
$$

We can use either Equation, (3-11), or Equation, (3-12), to evaluate $s_{r}$. For the former, we need, $s_{R}, R, \lambda$, and $r$. However, these are specified by Equations, (3-6), (2-16), (2-23), and column 3. The other alternative is to use Equation, (3-12). There, we need only, $s_{R}, T_{R}$, and the $T_{r}$ values specified under column 6. The $s_{R}$ and $T_{R}$ values are listed in Equations, (3-6), and, (3-2), respectively. These are alternative approaches to calculating the entropy density.

Equation, (3-11), makes explicit use of the shape parameter, full radius, and considered radius, $r$. The final expression is quite different than the one obtained for a truncated Gaussian (TG) distribution, obtained in reference [2]. There we had,

$$
s_{r} / s_{R}=(R / r)^{12 / 5} \exp \left[0.4\left(R^{2}-r^{2}\right) / \sigma^{2}\right]
$$

For that distribution, the shape parameters for the three black holes under consideration were evaluated, and the results were,

$$
\left(\sigma_{A}, \sigma_{B}, \sigma_{C}\right)=\left(1.951 \times 10^{2}, 1.992 \times 10^{3}, 2.241 \times 10^{8}\right) \mathrm{m} \quad(\mathrm{TG})
$$

Again, a different pdf leads to a different distribution of radiative mass, and other quantities within the black hole for the same size, or same total mass, black hole.

The next column entry in the tables is column 9, which relates to radiative force. By definition the radiative force is the radiative pressure at a particular $r$ value, multiplied by the surface area at the same radius, $r$. When looked at from the interior, this is a force acting inwards. When looked at from the outside, it is a force directed outwards. Per definition,

$$
F_{r}=F_{r}^{(4)}=p_{r}^{(4)} A_{r}^{(4)}=p_{r} A_{r}=p_{r} 2 \pi^{2} r^{3}
$$

Using this definition, and Equations, (3-10), with, (3-8), we can convince ourselves that

$$
F_{r} / F_{R}=p_{r} A_{r} /\left(p_{R} A_{R}\right)=\rho_{r} A_{r} /\left(\rho_{R} A_{R}\right)=f_{r} / f_{R}=\exp [\lambda(R-r)]
$$

For a particular value of $r$, we can evaluate the right hand side recognizing that both $\lambda$ and $R$ are known for the three black holes being analyzed. We can also easily evaluate $F_{R}$ on the left hand side, as this is just, $F_{R}=p_{R} A_{R}=p_{R} 2 \pi^{2} R^{3}$. The radii to be employed are as listed in Equations, (2-16). The radiative pressure at the surface, just inside the black hole, is specified by Equations, (3-4). Thus it is possible for us to determine, $F_{R}$, and using the right hand side of Equation, (3-16), the values for $F_{r}$, as well. These are the values worked out in column 9.

An alternative evaluation for $F_{r}$ would have been to multiply the column entries for pressure, given under column 5, with the corresponding surface areas, evaluated as, $A_{r}=2 \pi^{2} r^{3}$. This approach, however, which is Equation, (3-15), 
does not show us directly how the distribution and shape parameters come into play. Equation, (3-16), on the other hand, does. Equation, (3-16), can also be compared to its corresponding counterpart, where a truncated Gaussian is chosen as the pdf. This was worked out in reference [2], and we had

$$
F_{r} / F_{R}=f_{r} / f_{R}=\exp \left[\left(R^{2}-r^{2}\right) / 2 \sigma^{2}\right]
$$

The function on the right hand side is different, and thus the radiative force within the interior of the black hole has a different profile. The surface radiative force, $F_{R}$, however, has the same value for both distributions, and in fact, for all distributions.

We give three further columns in Tables 1-3. In column 10, we give the temperature gradient, $\mathrm{d} T_{r} / \mathrm{d} r$, as a function of 4 -D radius within the black hole. Under column 11, we list the specific heat, $C_{r} \equiv \mathrm{d} u_{r} / \mathrm{d} T_{r}$, as a function of radius. And finally, under column 12 , we calculate the internal energy gradient, $\mathrm{d} u_{r} / \mathrm{d} r$, within the black hole. We take each in turn.

We start with the results of Equations, (3-11), and (3-12). These can be equated to each other since the left hand sides are equal. From this we see that,

$$
T_{r} / T_{R}=(R / r)^{3 / 5} \mathrm{e}^{0.2 \lambda(R-r)}
$$

We differentiate this expression with respect to $r$, both left and right hand sides. We find that,

$$
\left(1 / T_{R}\right) \mathrm{d} T_{r} / \mathrm{d} r=\mathrm{e}^{0.2 \lambda(R-r)}\left[-0.2 \lambda(R / r)^{3 / 5}+R^{3 / 5}(-3 / 5) r^{-3 / 5}\right]
$$

We next divide the left hand side of Equation, (3-19), by the left hand side of Equation, (3-18), and do the same on the right hand side. The result is

$$
\left(1 / T_{r}\right) \mathrm{d} T_{r} / \mathrm{d} r=-0.2 \lambda-(3 / 5) r^{-8 / 5} r^{3 / 5}=-0.2 \lambda-3 /(5 r)
$$

Therefore,

$$
\mathrm{d} T_{r} / \mathrm{d} r=-(\lambda+3 / r) T_{r} / 5
$$

We know $\lambda$ and $T_{r}$ for each of the black holes. The exponential shape parameters, $\lambda$, are listed in Equations, (2-23). And the $T_{r}$ values are listed under column 7 in each of the tables. Therefore, with the help of Equation, (3-21), we can evaluate $\mathrm{d} T_{r} / \mathrm{d} r$ for selected values of radii, $r$. As mentioned, these values are tabulated under column 10 in Tables 1-3.

For a truncated Gaussian distribution, the counterpart to Equation, (3-21), was

$$
\mathrm{d} T_{r} / \mathrm{d} r=-\left(r / \sigma^{2}+3 / r\right) T_{r} / 5
$$

This was worked out in reference [2]. Upon comparing the two results, we notice that the second terms on the right hand side are equal. The first term differs due to the differing shape parameters.

For the $11^{\text {th }}$ column, we want to evaluate the quantity, $\mathrm{d} u_{r} / \mathrm{d} T_{r}$, which can be identified as the specific heat. To calculate this we make use of Equation, (3-9). We differentiate both left and right hand sides of this expression with respect to 
temperature, $T_{r}$. The result is

$$
\mathrm{d} u_{r}^{(4)} / \mathrm{d} T_{r}=5 a^{(4)} T_{r}^{4}=5 u_{r}^{(4)} / T_{r}=4 q_{r} / T_{r}=4 s_{r}
$$

Equations, (2-5b), and, (2-5c), have been used. The specific heat, therefore, in 4-D space, is equal to 4 times the entropy density, a simple result. We use the entries under entropy, column 8 , and multiply these by a factor of four to find, $C_{r}=\mathrm{d} u_{r} / \mathrm{d} T_{r}$. Those particular values are entered under column 11 in each of the tables.

For the last entries, column 12, we evaluate the internal energy density gradient, $\mathrm{d} u_{r} / \mathrm{d} r$. The simplest way to do that is to make use of the identity,

$$
\mathrm{d} u_{r} / \mathrm{d} r=\left(\mathrm{d} u_{r} / \mathrm{d} T_{r}\right)\left(\mathrm{d} T_{r} / \mathrm{d} r\right)=C_{r}\left(\mathrm{~d} T_{r} / \mathrm{d} r\right)
$$

We just determined both, $C_{r}$ and, $\mathrm{d} T_{r} / \mathrm{d} r$. These are given under columns 11 , and 10 , respectively. Therefore we multiply the entry in column 10 by the corresponding entry in column 11 to obtain the internal energy density gradient, $\mathrm{d} u_{r} / \mathrm{d} r$, at a particular radius. These drop off rather dramatically with increasing radius, as can be seen in Tables $1-3$, for the three black holes being analyzed.

\section{Radiative Mass, Gravitational Forces, and Entropy as Functions of Radius}

We have focused on radiative mass density, internal energy density, radiative pressure, heat density, entropy density, temperature, and radiative forces within the black hole. These held within specific layers inside the black hole. It is now time to consider cumulative effects. What is the total mass contained within an enclosing radius, $r$ ? What is the total internal energy, the total heat, the total gravitational force, the total entropy, etc. enclosed within radius, ? And what are the values at the surface, where $r=R$ ? It is now time to consider these questions. We gave an answer to these questions in a previous paper but these held for a truncated Gaussian distribution. We wish to formulate answers but ones, which now hold for a truncated exponential. The goal is to compare results, and see what the similarities and differences are.

We will follow the same format as in reference [2]. We investigate the same variables, and give similar tables, which will summarize our results. In the next section we will compare what we discover here with what was determined in paper [2], where we had a different pdf. Just like in the previous paper, we construct three Tables 4-6, at the end of the references. Table 4 holds for black hole A, which has one solar mass. Table 5 and Table 6 refer to black holes Table 2 and Table 3, respectively. They have masses 10 times, and $10^{6}$ times, the mass of the sun.

We start with the cumulative distribution function (cdf), defined by Equation, (2-10). For the pdf under consideration, the exponential distribution, the integral gives Equation, (2-19). We know the values for $R$ and $\lambda$ as these are fixed in Equations, (2-16), and, (2-23), for the three black holes under investigation. Therefore it is straightforward to calculate the cdf, $F_{r}^{C D F}$, using Equation, 
(2-19). The results are presented under column 2 in each of the Tables 4-6. Moreover, from Equation, (2-11), we also can calculate the radiative mass from $r=0$ up to, and including, radius, $r$. We simply multiply the total mass, which is given, by the appropriate cdf value. These results are presented under column 3 in each of the tables.

The radiative energy at radius, $r$, is also quite simple. We know that the radiative energy at radius $r$, between $r$ and $r+\mathrm{d} r$, must equal $\mathrm{d} E_{r}=e_{r} \mathrm{~d} V_{r}=e_{r} A_{r} \mathrm{~d} r=\rho_{r} c^{2} A_{r} \mathrm{~d} r$. The $\mathrm{d} V_{r}$ is the infinitesimal volume associated with that layer. This infinitesimal energy contribution can be integrated to find the radiative energy, up to, and including radius, $r$. The result is, using Equations, (2-13), (2-10), and (2-11),

$$
E_{r}=\int_{0}^{r} \rho_{r} c^{2} A_{r} \mathrm{~d} r=M_{R} c^{2} \int_{0}^{r} f_{r} \mathrm{~d} r=M_{r} c^{2}
$$

All quantities are 4-D, and we have dispensed with the superscripts (4) over the individual entities. As expected, the total radiative energy up to a particular radius is simply, $M_{r} c^{2}$.

The total radiative energy is not entered under any specific column. Rather, we focus on the constituent parts, which are the internal energy, $U_{r}$, the heat energy, $Q_{r}$, and the work done against radiative pressure, $W_{r}$, which has to be contained by gravity. The sum of these three contributions will give us, $E_{r}=M_{r} c^{2}$. In 4-D space we found that [2],

$$
U_{r}=\frac{4}{10} M_{r} c^{2}, Q_{r}=\frac{1}{2} M_{r} c^{2}, W_{r}=\frac{1}{10} M_{r} c^{2}
$$

The factors of $\frac{4}{10}, \frac{5}{10}$, and, $\frac{1}{10}$, in all these relations remain the same, whether we consider, a layer, an enclosing volume, or the total volume. The factors are imposed upon us by the dimensionality of space and the fact that we are dealing with blackbody radiation. What is obvious with these relations is the fact that the internal energy makes up $40 \%$ of the total energy, the trapped heat represents $50 \%$ of the total energy, and the work done against pressure makes up $10 \%$ of the total. In $3-\mathrm{D}$ space, the proportions would be different. In three dimensions, the corresponding proportions would be, $\frac{3}{8}=37.5 \%, \frac{4}{8}=50 \%$, and, $\frac{1}{8}=12.5 \%$.

The values for, $U_{r}, Q_{r}$, and, $W_{r}$, are entered under columns 4, 5, and, 6, respectively in each of the Tables 4-6. Again their sum equals, $E_{r}=M_{r} c^{2}$, as we are assuming that all contributions make up the mass of the black hole. What holds the black hole together under radiative forces, which wants to blow it out, is gravity. This provides the surface tension necessary to contain the enclosed radiation. As was shown in a previous work, the 4-D hydrostatic equation reads

$$
\mathrm{d} p_{r}^{(4)} / \mathrm{d} r=-G_{r}^{(4)} M_{r}^{(4)} \rho_{r}^{(4)} / r^{3}-3 p_{r}^{(4)} / r
$$

The net radiative pressure pushing out is balanced by the gravitational force 
pulling in. The second term on the right hand side takes into account the change in areas between the upper part of the layer and the lower part. Equation, (4-3), holds for each layer making up the 4-D black hole. See references [2] for more details.

We next focus on the gravitational force. Equation, (4-3), will be our starting point. We know from Equations, (3-10), and, (3-8), that

$$
p_{r} A_{r} /\left(p_{R} A_{R}\right)=\rho_{r} A_{r} /\left(\rho_{R} A_{R}\right)=\mathrm{e}^{\lambda(R-r)}
$$

We take the derivative of the first and last part of this equation with respect to radius, and find

$$
\left(\mathrm{d} p_{r} / \mathrm{d} r A_{r}+p_{r} \mathrm{~d} A_{r} / \mathrm{d} r\right) /\left(p_{R} A_{R}\right)=-\lambda \mathrm{e}^{\lambda(R-r)}
$$

We next divide the left hand side of Equation, (4-5), by the left hand side of Equation, (4-4). We do the same for the right hand side. This allows us to write,

$$
\left(1 / p_{r} \mathrm{~d} p_{r} / \mathrm{d} r+1 / A_{r} \mathrm{~d} A_{r} / \mathrm{d} r\right)=-\lambda
$$

However, in 4-D space, $A_{r}=2 \pi^{2} r^{3}$, and thus, $\mathrm{d} A_{r} / \mathrm{d} r=6 \pi^{2} r^{2}$. Therefore, we can simplify Equation, (4-6), to

$$
\mathrm{d} p_{r} / \mathrm{d} r=-(\lambda+3 / r) p_{r}
$$

This we substitute into Equation, (4-3), to obtain

$$
-(\lambda+3 / r) p_{r}=-G_{r}^{(4)} M_{r} \rho_{r} / r^{3}-3 p_{r} / r
$$

The second terms on both left and right hand sides cancel. Our next step is to recognize that,

$$
\rho_{r}=e_{r} / c^{2}=10 p_{r} / c^{2}
$$

The second equality in Equation, (2-4), has been utilized to obtain the final equality. We also substitute this expression into Equation, (4-8), in order to eliminate the $\rho_{r}$ term. This allows us to write, after we cancel the $p_{r}$ term,

$$
G_{r}^{(4)} M_{r}=0.1 c^{2} \lambda r^{3}
$$

This is a remarkably simple expression, and shows us that the 4-D gravitational "constant" is not a constant. In fact, this expression is quite similar to that obtained for the truncated Gaussian distribution in reference [2]. There we had, as a corresponding expression,

$$
G_{r}^{(4)} M_{r}=0.1 c^{2} r^{4} / \sigma^{2}
$$

We notice a certain degree of similarity. The difference is in the power law. In Equation, (4-10), $G_{r}^{(4)} M_{r}$ is proportional to $r^{3}$, whereas Equation, (4-11), it is proportional to $r^{4}$, for a given size (mass) black hole. The $G_{r}^{(4)} M_{r}$ values, as calculated in Equation, (4-10), are tabulated under column 7 for each of the black holes under consideration, in Tables 4-6. Also listed in the tables, under column 8, are the $G_{r}^{(4)}$ values as calculated using Equation, (4-10). It is clearly seen that $G_{r}^{(4)}$ varies with $4-\mathrm{D}$ radius, which seems to be a feature of 4-D space. The units are also different.

We specialize Equation, (4-10), to $r=R$. At the event horizon, 


$$
G_{R}^{(4)} M_{R}=0.1 c^{2} \lambda R^{3}
$$

Using the Schwarzschild relation, $R=2 G M_{R} / c^{2}$, this equation can be recast in the form,

$$
G_{R}^{(4)}=0.2 G \lambda R^{2}
$$

There will be a sharp discontinuity in the gravitational constant from its 3-D value, $G$, which is Newton's constant, to a new value, $G_{R}^{(4)}$, upon entering 4-D space. It will turn out that, $0.2 \lambda R^{2} \neq 1$. From the tables, it is seen that, $G_{R}^{(4)} \gg G$. We have listed $G_{r}^{(4)}$ under column 8. This drastic and abrupt increase in gravitational coupling "constant" when entering 4-D space has to do with the discontinuity of space itself. Even though the radius does not change, the surface area, and volume do. Therefore many quantities upon entry into the 4-D black hole experience a sharp discontinuity. The temperature decreases abruptly. So do the internal energy density, the radiative pressure, the heat density, etc. We referred to this as our waterfall model, and the details are explained in reference [1].

For the truncated Gaussian distribution, detailed in reference [2], we had a similar state of affairs. We found that at, $r=R$, the counterparts to Equations, (4-12), and (4-13), are,

$$
G_{R}^{(4)} M_{R}=0.1 c^{2} R^{4} / \sigma^{2} \quad(\mathrm{TG})
$$

And,

$$
G_{R}^{(4)}=0.2 G R^{3} / \sigma^{2} \quad(\mathrm{TG})
$$

Except for the power law, we see a certain similarity between these Equations, and Equations, (4-12), and, (4-13).

We next derive a surprising result. As shown in reference [2], utilizing a 4-D version of Gauss' law, we saw, quite generally, that the gravitational acceleration within the black hole, reduced to, $g_{r}=-G_{r}^{(4)} M_{r} / r^{3}$. Spherical symmetry was assumed in 4-D space. If we substitute Equation, (4-10), into this expression, we obtain,

$$
g_{r}=-G_{r}^{(4)} M_{r} / r^{3}=-0.1 c^{2} \lambda=\mathrm{a} \text { constant } \quad(\mathrm{TE})
$$

A test particle within the 4-D black hole will thus experience the same gravitational force, irrespective of radius! Moreover, the exponential shape parameter, which depends only on the mass, or size, of the black hole, will determine its numerical strength. This holds true only for the truncated exponential distribution. Even though the acceleration due to gravity is a constant for the truncated exponential distribution (TE), we have listed them under column 9 for each black hole being analyzed in Tables 4-6. This is to emphasize a point, but also to show the differences between the various masses.

For the truncated Gaussian distribution, we had quite another result. There, we obtained,

$$
g_{r}=-G_{r}^{(4)} M_{r} / r^{3}=-0.1 c^{2} r / \sigma^{2}
$$


which is proportional to, $r$, for a given size (mass) black hole. The gravitational acceleration increased linearly with radius in 4-D space, even though the radiative mass density was not a constant. What we see with these two examples is that the pdf chosen will determine the gravitational "packing" within the black hole, i.e., how radiative mass is stored, and contained within the black hole.

We have already mentioned that at the 3-D/4-D interface, which is at the surface, or event horizon, of the black hole in our model, we can expect discontinuities. This holds true for the gravitational acceleration as well. We consider the gravitational acceleration at the event horizon, where $r=R$. Using Equations, (4-16), and, (4-17), we notice that

$$
g_{R}=-0.1 c^{2} \lambda \quad(\mathrm{TE}) g_{R}=-0.1 c^{2} R / \sigma^{2}
$$

These values can be compared to the acceleration due to gravity on the 3-D side. There we would obtain, $g_{R}^{(3)}=-G M_{R} / R^{2}=-0.5 c^{2} / R$, where in the second equality, we have used Schwarzschild relation. It is clear that all three values are different, and unique. None can be made to equal the other, and so, we have a discontinuity.

We next turn to the gravitational potential, $\varphi_{r}$. This is a four dimensional quantity which depends on radius, $r$. The defining equation is

$$
\varphi_{r}-\varphi_{0}=-\int_{0}^{r} g_{r} \mathrm{~d} r
$$

The constant of integration will be fixed by setting the gravitational potential in 4-D space equal to that in 3-D space at the event horizon. In this way we can guarantee for the same equipotential surface in both spaces.

For the truncated exponential distribution, we saw that the gravitational acceleration within a specific black hole is just a constant. Refer to Equation, (4-16). The integral thus becomes trivial, and we obtain,

$$
\varphi_{r}-\varphi_{0}=\left(0.1 c^{2}\right)(\lambda r)
$$

This becomes, at radius, $R$,

$$
\varphi_{r}-\varphi_{0}=\left(0.1 c^{2}\right)(\lambda R)
$$

We demand that, $\varphi_{R}^{(4)}=\varphi_{R}^{(3)}=-G M_{R} / R$. This gives

$$
\varphi_{0}+\left(0.1 c^{2} \lambda R\right)=-G M_{R} / R=-0.5 c^{2}
$$

For the last equality, the Schwarzschild relation was used. Therefore, putting all this together, we obtain for the constant of integration,

$$
\varphi_{0}=-c^{2}(0.5+0.1 \lambda R)=-G M_{R} / R(1+0.2 \lambda R)
$$

This we substitute into Equation, (4-20), and simplify. The final result for the gravitational potential is thus,

$$
\varphi_{r}=\varphi_{0}+0.1 c^{2} \lambda r=-G M_{R} / R[1+0.2 \lambda(R-r)]
$$

We note that at, $r=R$, we obtain the three dimensional result. Also, as the radius decreases, the gravitational potential becomes more and more negative.

Equation, (2-24), can be compared to the corresponding truncated Gaussian 
result, worked out in reference [2]. There we found that

$$
\varphi_{r}=\left(-G M_{R} / R\right)\left[1+0.1\left(R^{2}-r^{2}\right) / \sigma^{2}\right] \quad(\mathrm{TG})
$$

Upon comparing results, there is a certain similarity in that solutions match at the 3-D/4-D interface, and the gravitational potential becomes more and more negative as the radius decreases. But there the similarity ends as we have, in Equation, (4-24), an entirely different function.

We have tabulated the values for the gravitational potential, using Equation, (4-24), for various radii. These values are given under column 10, in Tables 4-6. All that was needed were the total radii, and the shape parameters, for each of the black holes we are investigating. These values were specified in Equations, (2-16), and, (2-23).

We have two more column entries, columns 11 and 12. Under column 11 we calculate the gravitational force at particular radii, and under column 12, we give the entropy, also as a function of radius. We first consider the 4-D gravitational force. Within a layer between, $r$, and, $r+\mathrm{d} r$, we have a well-defined gravitational force acting on that layer,

$$
\mathrm{d} F_{G, r}=\left(g_{r} \mathrm{~d} M_{r}\right)=\left(-0.1 c^{2} \lambda\right)\left(\mathrm{d} M_{r}\right)
$$

Equation, (4-16), was used. To find the total gravitational force at a specific radius, we integrate this expression, and find,

$$
F_{G, r}=\int_{0}^{r}-0.1 c^{2} \lambda \mathrm{d} M_{r}=-0.1 c^{2} \lambda M_{r}
$$

From this equation, we see that the gravitational force at radius, $r$, is simply proportional to the radiative energy enclosed within that radius, $M_{r} c^{2}$. The shape parameter determines the constant of proportionality. That the gravitational force should be proportional to $M_{r}$ is not surprising, but that it depends on little else is. In fact, a force which is proportional to stored energy is very unusual. The gravitational force is listed under column 11 in Tables 4-6. We only need the $M_{r}$ values, given under column 2, and the shape parameters, specified by Equations, (2-16).

For a truncated Gaussian or Normal distribution, the corresponding expression for the gravitational force was somewhat different. There we needed the 4-D radiative force at the surface, $F_{R}$. The expression for the gravitational force was [2],

$$
\begin{aligned}
F_{G, r} & =\int_{0}^{r}\left(g_{r}\right)\left(\rho_{r} A_{r} \mathrm{~d} r\right)=\int_{0}^{r}\left(-0.1 c^{2} r / \sigma^{2}\right)\left(M_{R} f_{r} \mathrm{~d} r\right) \\
& =\left(-0.1 M_{R} c^{2}\right) \int_{0}^{r}\left(r / \sigma^{2}\right)\left(f_{r} \mathrm{~d} r\right) \\
& =\left(-0.1 M_{R} c^{2}\right) \int_{0}^{r}\left(r / \sigma^{2}\right)\left(f_{R} \exp \left[\frac{R^{2}-r^{2}}{2 \sigma^{2}}\right] \mathrm{d} r\right) \\
& =\left(-0.1 M_{R} c^{2} f_{R}\right) \int_{0}^{r}\left(r / \sigma^{2}\right)\left(\exp \left[\frac{R^{2}-r^{2}}{2 \sigma^{2}}\right] \mathrm{d} r\right) \\
& =-F_{R}\left[\mathrm{e}^{R^{2} / 2 \sigma^{2}}-\mathrm{e}^{\left(R^{2}-r^{2}\right) / 2 \sigma^{2}}\right]
\end{aligned}
$$


As stated, $F_{R}=p_{1}^{(4)} A_{R}^{(4)}$, is the 4-D radiative force just inside the black hole, at radius, $R$. This radiative force can be calculated. We find, using the arguments of reference [2],

$$
\begin{aligned}
F_{R} & =F_{R}^{(4)}=p_{1}^{(4)} A_{R}^{(4)}=0.8 p_{2}^{(3)} A_{R}^{(3)} \\
& =0.8 u_{2}^{(3)} / 3 \times 4 \pi R^{2} \\
& =32 \pi / 30 a^{(3)}(2.725)^{4} R^{2} \\
& =16 \pi / 15\left(7.5657 \times 10^{-16}\right)(2.725)^{4} R^{2} \\
& =1.398 \times 10^{-13} R^{2}
\end{aligned}
$$

Equation, (4-29), holds for an isolated static black hole (ISBH), and all quantities are in the MKS system of units. Using Equation, (4-29), we can determine the gravitational force, in Equation, (4-28). Even though Equation, (4-29), holds technically only for an isolated, static black hole, it can also be applied with a new temperature, $T_{2}>2.725 \mathrm{~K}$, for a dynamic situation, when there is net inflow. The point is that the gravitational force can be calculated in terms of the radiative force, in either scenario.

We next compare the gravitational forces at the event horizon, where, $r=R$, for both the exponential distribution and the Gaussian distribution. We focus on a static situation. For the truncated exponential distribution, we use Equation, (4-27), and for the truncated Gaussian distribution, we use Equation, (4-28), with Equation, (4-29). For the former we obtain,

$$
F_{G, R}=-0.1 M_{R} c^{2} \lambda
$$

For the latter, we find,

$$
F_{G, R}=-F_{R}\left[\mathrm{e}^{R^{2} / 2 \sigma^{2}}-1\right]
$$

We evaluate numerically both sets of values for each of the black holes under consideration. We have the radii specified in Equations, (2-16). The $\lambda$ values are indicated in Equations, (2-23). We also have the $\sigma$ values specified for each of the black holes in Equations, (3-14). Numerically, the results are,

$$
\left(F_{G, R}^{A}, F_{G, R}^{B}, F_{G, R}^{C}\right)=\left(7.07 \times 10^{44}, 6.79 \times 10^{44}, 5.38 \times 10^{44}\right) \text { Newtons }
$$

These forces are for the truncated exponential. For the truncated Gaussian, the corresponding calculations give,

$$
\left(F_{G, R}^{A}, F_{G, R}^{B}, F_{G, R}^{C}\right)=\left(7.30 \times 10^{43}, 7.16 \times 10^{45}, 6.36 \times 10^{43}\right) \text { Newtons }
$$

Upon comparing these two sets of results, we notice that they are comparable in value.

Finally, let us consider the entropy. There are many ways to calculate the entropy, $S_{r}=S_{R}^{(4)}$, as a function of radius. Perhaps the most direct way is to use Equation, (3-11). First, we recognize that within a particular layer, between $r$ and, $r+\mathrm{d} r$, the entropy amounts to, $\mathrm{d} S_{r}=s_{r} \mathrm{~d} V_{r}=s_{r} A_{r} \mathrm{~d} r$, where, $A_{r}=2 \pi^{2} r^{3}$, is the 4-D surface area. If we multiply Equation, (3-11), by $A_{r} / A_{R}$, we obtain 


$$
s_{r} A_{r} /\left(s_{R} A_{R}\right)=(r / R)^{3}(R / r)^{12 / 5} \mathrm{e}^{0.8 \lambda(R-r)}=(r / R)^{3 / 5} \mathrm{e}^{0.8 \lambda(R-r)}
$$

We next integrate both the left and right hand sides over $\mathrm{d} r$. The result is,

$$
\begin{gathered}
\int_{0}^{r} S_{r} A_{r} /\left(s_{R} A_{R}\right) \mathrm{d} r=\int_{0}^{r}(r / R)^{3} \mathrm{e}^{0.8 \lambda(R-r)} \mathrm{d} r \\
1 /\left(s_{R} A_{R}\right)\left[S_{r}-S_{0}\right]=-(5 / R)^{\frac{3}{5}}\left(\frac{1}{4 \lambda}\right)^{\frac{8}{5}}\left[5 \Gamma\left(\frac{8}{5}, 0.8 \lambda r\right)-3 \Gamma\left(\frac{3}{5}\right)\right] \mathrm{e}^{0.8 \lambda R}
\end{gathered}
$$

In this equation, $\Gamma(s, x)$, is the upper incomplete gamma function, and, $\Gamma(s)$, is the regular gamma function. We set our constant of integration, $S_{0}$, equal to zero since, at $r=0$, we can assume no entropy.

We next multiply Equation, (4-35), through by $s_{R} A_{R}$, and simplify to obtain,

$$
S_{r}=-S_{R} 2 \pi^{2} R^{12 / 5}(5 / 4 \lambda)^{8 / 5}\left[\Gamma\left(\frac{8}{5}, 0.8 \lambda r\right)-\frac{3}{5} \Gamma\left(\frac{3}{5}\right)\right] \mathrm{e}^{0.8 \lambda R}
$$

We have values for, $s_{R}, R$, and, $\lambda$, for each of the black holes being analyzed. Refer to Equations, (3-6), (2-16), and, (2-23), respectively. Therefore, $S_{r}$, can be evaluated as a function of radius for each of the black holes in question. We have listed the $S_{r}$ entries under column 12 in Tables 4-6, for the specific radii indicated under column 1 .

We can specialize to, $r=R$, and compare these values for entropy. We find, upon referring to the last row in Tables 4-6, that the following values for total entropy hold.

$$
\left(S_{R}^{A}, S_{R}^{B}, S_{R}^{C}\right)=\left(4.01 \times 10^{36}, 1.65 \times 10^{40}, 1.99 \times 10^{46}\right) \text { Joules } / \mathrm{K}
$$

These values are very low when compared to the Bekenstein entropy [15] [16] [17], just as in the truncated Gaussian case. This would seem to suggest that, if this model is correct, the black hole is a highly ordered state. This is very contrary to what is commonly thought.

We have calculated the entropy using our truncated Gaussian distribution. As seen in reference [2], we obtained,

$$
\begin{aligned}
S_{r} & =\left(-F_{R} / T_{R}\right) R^{-\frac{3}{5}}(5 / 2)^{\frac{9}{5}}\left[\Gamma\left(\frac{4}{5}, \frac{2 r^{2}}{5 \sigma^{2}}\right)-\Gamma\left(\frac{4}{5}\right)\right] \sigma^{8 / 5} \mathrm{e}^{\frac{2 R^{2}}{5 \sigma^{2}}} \\
& =\left(+F_{R} / T_{R}\right) R^{-\frac{3}{5}}(5 / 2)^{\frac{9}{5}} \gamma\left(\frac{4}{5}, \frac{2 r^{2}}{5 \sigma^{2}}\right) \sigma^{8 / 5} \mathrm{e}^{\frac{2 R^{2}}{5 \sigma^{2}}}
\end{aligned}
$$

here, $\gamma(s, x)=\Gamma(s)-\Gamma(s, x)>0$, is the lower incomplete gamma function. We notice that the term within the brackets on the right hand side of Equation, (4-36), involving the gamma functions, cannot be simplified. But here, in Equation, (4-38), the terms can be combined. Both Equations, (4-36), and, (4-38), are rather complicated, but they both give values for the total entropy, which are low. Perhaps black holes are indeed, highly ordered objects. The counterpart to Equation, (4-37), is

$$
\left(S_{R}^{A}, S_{R}^{B}, S_{R}^{C}\right)=\left(1.63 \times 10^{37}, 6.59 \times 10^{40}, 7.24 \times 10^{46}\right) \text { Joules } / \mathrm{K}
$$


This holds for a truncated Gaussian, and these results are obtained using Equation, (4-38). When compared to Equation, (4-37), we see that these values are comparable. They are however, definitely larger in value. It is well known that the truncated Gaussian has the maximum mathematical entropy of all probability distribution functions. This holds true with support, $r \in[0, R]$, or without.

\section{Discussion of Results}

Now that the values have been evaluated, and entered into the tables, it is time to compare results. We start with Tables $1-3$. We consider values at $r=0.01 R$, and compare those values to the corresponding quantities at the surface. The radius, $r=0.01 R$, is close to the core, and it will be insightful to compare those values to surface values. We will also give, right next to the truncated exponential values, the corresponding truncated Gaussian values. This will enable us to make further comparisons.

We start with temperature. Ultimately, for a black body, everything depends on temperature. This includes the internal energy density, the radiative pressure, the radiative forces, the heat density, the entropy density, etc.. These quantities are, by and large, some factor times the temperature taken to the fifth power. Entropy density is an exception, as well as radiative force. The temperature, $T_{0.01 R}$, holds at $r=0.01 R$, whereas, $T_{1}=T_{R}$, is the temperature at the surface, just inside the black hole. The superscripts A, B, and, C, refer to the three specific black holes under consideration. The corresponding Tables 1-3. Resorting to the tables, we find (all temperatures are in $\mathrm{K}$ )

$$
\begin{aligned}
& {\left[T_{0.01 R}^{A}, T_{R}^{A}\right]=\left[2.05 \times 10^{10}, 0.115\right]} \\
& {\left[T_{0.01 R}^{B}, T_{R}^{B}\right]=\left[5.16 \times 10^{9}, 7.26 \times 10^{-2}\right](\mathrm{T})} \\
& {\left[T_{0.01 R}^{C}, T_{R}^{C}\right]=\left[5.16 \times 10^{6}, 7.26 \times 10^{-3}\right]}
\end{aligned}
$$

We see very clearly that the smaller the black hole (lessor mass), the higher the core temperature. Equations, (5-1), can be compared to the corresponding values for a truncated Normal distribution. These values were worked out in reference [2], and referring to those tables, we had

$$
\begin{aligned}
& {\left[T_{0.01 R}^{A}, T_{R}^{A}\right]=\left[1.64 \times 10^{10}, 0.115\right]} \\
& {\left[T_{0.01 R}^{B}, T_{R}^{B}\right]=\left[4.11 \times 10^{9}, 7.26 \times 10^{-2}\right](\mathrm{TG})} \\
& {\left[T_{0.01 R}^{C}, T_{R}^{C}\right]=\left[4.02 \times 10^{6}, 7.26 \times 10^{-3}\right]}
\end{aligned}
$$

The surface temperatures are the same as in Equations, (5-1). The core temperatures are comparable, but less than in relations, (5-2). The least massive black holes have the highest core temperatures.

Another quantity we focus on as it relates to Tables 1-3, is radiative force, $F_{r}$. The radiative force, $F_{0.01 R}$, will refer to the radiative force at, $r=0.01 R$, whereas, $F_{R}$, is the value at the surface. The superscripts refer to the black holes being analyzed. The radiative force can be calculated, using, 
$F_{r}=p_{r} A_{r}=p_{r} 2 \pi^{2} r^{3}$. The pressure values are entered under column 4 , and we multiply the pressure by the 4-D surface area to find the $F_{r}$ values. We find

$$
\begin{aligned}
& {\left[F_{0.01 R}^{A}, F_{R}^{A}\right]=\left[2.20 \times 10^{44}, 1.22 \times 10^{-6}\right]} \\
& {\left[F_{0.01 R}^{B}, F_{R}^{B}\right]=\left[2.22 \times 10^{44}, 1.22 \times 10^{-4}\right](\mathrm{TE})} \\
& {\left[F_{0.01 R}^{C}, F_{R}^{C}\right]=\left[2.22 \times 10^{44}, 1.22 \times 10^{+6}\right]}
\end{aligned}
$$

All forces are measured in Newtons. As is plain to see, the radiative forces are close to zero at the surface but quite substantial at the core. In fact, at the core, they have almost identical values.

Equations, (5-3), can be compared to the corresponding values for a truncated Normal distribution. Those values are found in the same manner, and referring to the tables in reference [2], we obtain,

$$
\begin{aligned}
& {\left[F_{0.01 R}^{A}, F_{R}^{A}\right]=\left[7.23 \times 10^{43}, 1.22 \times 10^{-6}\right]} \\
& {\left[F_{0.01 R}^{B}, F_{R}^{B}\right]=\left[7.08 \times 10^{43}, 1.22 \times 10^{-4}\right](\mathrm{TG})} \\
& {\left[F_{0.01 R}^{C}, F_{R}^{C}\right]=\left[6.31 \times 10^{43}, 1.22 \times 10^{+6}\right]}
\end{aligned}
$$

The surface radiative forces are the same as in Equations, (5-3). The core values are likewise very close to each other, irrespective of the size (mass) of the black hole. The core radiative forces in Equations, (5-4), are less than those indicated in Equations, (5-3).

We next proceed to Tables 4-6. These quantities are cumulative in nature, i.e., they hold from, $r=0$, up to, and including, some finite value for $r$. We start with radiative mass. Using the same conventions as before, and referring to the Tables 4-6, we find,

$$
\begin{aligned}
& {\left[M_{0.01 R}^{A}, M_{R}^{A}\right]=\left[1.37 \times 10^{30}, 1.99 \times 10^{30}\right]} \\
& {\left[M_{0.01 R}^{B}, M_{R}^{B}\right]=\left[1.34 \times 10^{31}, 1.99 \times 10^{31}\right](\mathrm{TE})} \\
& {\left[M_{0.01 R}^{C}, M_{R}^{C}\right]=\left[1.17 \times 10^{36}, 1.99 \times 10^{36}\right]}
\end{aligned}
$$

These masses are in, kg. Upon comparing the core values to the surface values, we notice something surprising. The core values are exceedingly large in relation to the total mass. Within one percent of the radius, we already have the following proportions of the entire (total) mass.

$$
(1.37 / 1.99,1.34 / 1.99,1.17 / 1.99)=(69 \%, 67 \%, 59 \%)(\mathrm{TE})
$$

These proportions are quite high but then we are dealing with an exponential distribution, where the concentration is very near the center. This is the distribution which comes closest to mimicking a singularity at the center. It comes close to being singular but still is finite at, $r=0$, as can be seen from equation, (2-18). If we set $r=0$ in this equation, then, $f_{0}=\lambda /\left(1-\mathrm{e}^{-\lambda R}\right)$. Even though this value is large, it is not infinite.

The truncated Normal distribution does not come close to packing/concentrating the radiative mass (energy) within such a small volume. As can be seen by referring to the tables in reference [2], we have 


$$
\begin{aligned}
& {\left[M_{0.01 R}^{A}, M_{R}^{A}\right]=\left[2.39 \times 10^{29}, 1.99 \times 10^{30}\right]} \\
& {\left[M_{0.01 R}^{B}, M_{R}^{B}\right]=\left[2.35 \times 10^{30}, 1.99 \times 10^{31}\right](\mathrm{TG})} \\
& {\left[M_{0.01 R}^{C}, M_{R}^{C}\right]=\left[2.09 \times 10^{35}, 1.99 \times 10^{36}\right]}
\end{aligned}
$$

These values are decidedly less. Here the corresponding proportions are,

$$
(0.239 / 1.99,0.235 / 1.99,0.209 / 1.99)=(12 \%, 12 \%, 11 \%)(\mathrm{TG})
$$

These percentages are still, surprisingly, relatively high, indicating large packing within the interior, but they are not as high as in Equations, (5-6).

Another series of entries in Tables 4-6, deal with the gravitational "constant" in 4-D space. As we can clearly see, these values are not constant, but radius dependent. See Equation, (4-10). For the truncated Gaussian distribution, we had a similar state of affairs, i.e., $G_{r}^{(4)}$ was a function of radius. There the evaluation rests on relation, (4-11). Moreover, in both instances, when we transition from 3-D space, where $G$ is a constant, to 4 -D space where it is no longer a constant, we find a jump or discontinuity in value. We obtain a dramatic increase in the gravitational coupling constant. We have found this condition for many variables because in transitioning from three-dimensional space, to 4-D space, there is a break in spatial dimension. In reference [1], this was referred to as a "waterfall" model.

We first give the gravitational coupling in 4-D space using our truncated exponential distribution. Using the same notation as before, and referring to the Tables 4-6, we find,

$$
\begin{aligned}
& {\left[G_{0.01 R}^{A}, G_{R}^{A}\right]=\left[6.69 \times 10^{-12}, 4.61 \times 10^{-6}\right]} \\
& {\left[G_{0.01 R}^{B}, G_{R}^{B}\right]=\left[6.56 \times 10^{-11}, 4.43 \times 10^{-5}\right](} \\
& {\left[G_{0.01 R}^{C}, G_{R}^{C}\right]=\left[5.95 \times 10^{-6}, 3.51 \times 10^{0}\right]}
\end{aligned}
$$

The units are different than their three dimensional counterpart, $G=6.67 \times 10^{-11}$ Newtons $\cdot \mathrm{m}^{2} / \mathrm{kg}^{2}$. In 4-D space, $G_{r}=G_{r}^{(4)}$ is measured in, Newtons $\cdot \mathrm{m}^{3} / \mathrm{kg}^{2}$. We notice that, at the event horizon, we have a sharp increase in gravitational coupling upon entering the $4-\mathrm{D}$ space. Second, the gravitational coupling decreases substantially as one enters the black holes interior. Thirdly, the largest, most massive, black holes have the largest gravitational coupling constants. And finally, upon entering 4-D space, the units change.

We compare the results in Equations, (5-9), with the corresponding values for a truncated Gaussian distribution. These were worked out in reference [2], and we repeat them here in order to make comparisons. The values obtained there were

$$
\begin{aligned}
& {\left[G_{0.01 R}^{A}, G_{R}^{A}\right]=\left[7.51 \times 10^{-13}, 9.04 \times 10^{-6}\right]} \\
& {\left[G_{0.01 R}^{B}, G_{R}^{B}\right]=\left[7.36 \times 10^{-12}, 8.67 \times 10^{-5}\right](\mathrm{TG})} \\
& {\left[G_{0.01 R}^{C}, G_{R}^{C}\right]=\left[6.53 \times 10^{-7}, 6.85 \times 10^{0}\right]}
\end{aligned}
$$

Again, we notice the discontinuous increase in gravitational coupling between 
masses upon entry from 3-D space. Again, the gravitational coupling decreases as one enters the interior. And, as before, the largest, most massive black holes have the strongest gravitational couplings. The units for gravitational coupling also change when entering 4-D space from 3-D space. The values here depend on the Gaussian shape parameter, $\sigma$. Comparatively, the results in equations, (5-9), and, (5-10), are quite similar, although Equations, (5-10), have somewhat stronger values at the surface. Close to the core, however, the exponential distribution gives larger $G_{r}$ values. This comes as no surprise because the mass concentration is more intense there.

Next, we look at the gravitational acceleration. For a truncated exponential distribution, the surprising result is that it is a constant throughout the black hole. Therefore, referring to the Tables 4-6, we find that

$$
\begin{aligned}
& {\left[g_{0.01 R}^{A}, g_{R}^{A}\right]=\left[-3.56 \times 10^{14},-3.56 \times 10^{14}\right]} \\
& {\left[g_{0.01 R}^{B}, g_{R}^{B}\right]=\left[-3.41 \times 10^{13},-3.41 \times 10^{13}\right]} \\
& {\left[g_{0.01 R}^{C}, g_{R}^{C}\right]=\left[-2.71 \times 10^{8},-2.71 \times 10^{8}\right]}
\end{aligned}
$$

For black holes with larger mass, the gravitational acceleration does decrease. However, for a specific mass, this value is uniform. We compare this to the corresponding values if one were to use a truncated Normal distribution instead. Employing the tables in reference [2], we notice that

$$
\begin{aligned}
& {\left[g_{0.01 R}^{A}, g_{R}^{A}\right]=\left[-6.97 \times 10^{12},-6.97 \times 10^{14}\right]} \\
& {\left[g_{0.01 R}^{B}, g_{R}^{B}\right]=\left[-6.69 \times 10^{11},-6.69 \times 10^{13}\right]} \\
& {\left[g_{0.01 R}^{C}, g_{R}^{C}\right]=\left[-5.28 \times 10^{6},-5.28 \times 10^{8}\right]}
\end{aligned}
$$

Here, obviously, the values are not constant within the black hole. The gravitational acceleration does decrease, as one decreases the 4-D radius. When Equation, (5-12), is compared to Equation, (5-11), we see that the values at the surface are comparable. However within the interior, we have far lessor values for gravitational acceleration if the distribution is Gaussian versus exponential. For the truncated Gaussian, the gravitational acceleration is proportional to radius, $r$, as can be seen by Equation, (4-17).

We focus on two more sets of entries. The first is the gravitational force, $F_{G, r}$, at radius $r$.

Referring to the Tables 4-6, we see that

$$
\begin{aligned}
& {\left[F_{G, 0.01 R}^{A}, F_{G, R}^{A}\right]=\left[4.88 \times 10^{44}, 7.07 \times 10^{44}\right]} \\
& {\left[F_{G, 0.01 R}^{B}, F_{G, R}^{B}\right]=\left[4.58 \times 10^{44}, 6.79 \times 10^{44}\right](\mathrm{TE})} \\
& {\left[F_{G, 0.01 R}^{C}, F_{G, R}^{C}\right]=\left[3.17 \times 10^{44}, 5.38 \times 10^{44}\right]}
\end{aligned}
$$

All values are in, Newtons. Looking at these values, we notice that there is very little variation. In fact, in terms of percentages, the core values are a substantial part of the total gravitational force at the surface. We have

$$
(4.88 / 7.07,4.58 / 6.79,3.17 / 5.38)=(69 \%, 67 \%, 59 \%)(\mathrm{TE})
$$


These are the same percentages as in Equations, (5-6), which hold for the radiative mass. That the two are proportional is not surprising. That the core values are a high percentage of the total is. The gravitational force at the surface is a major contribution to the surface tension, as shown in reference [1].

We compare this to the corresponding expressions for the truncated normal distribution. Referring to the Tables 4-6, in reference [2], we find that

$$
\begin{aligned}
& {\left[F_{G, 0.01 R}^{A}, F_{G, R}^{A}\right]=\left[8.32 \times 10^{41}, 7.30 \times 10^{43}\right]} \\
& {\left[F_{G, 0.01 R}^{B}, F_{G, R}^{B}\right]=\left[7.83 \times 10^{43}, 7.16 \times 10^{45}\right](\mathrm{TG})} \\
& {\left[F_{G, 0.01 R}^{C}, F_{G, R}^{C}\right]=\left[5.50 \times 10^{41}, 6.36 \times 10^{43}\right]}
\end{aligned}
$$

With the exception of black hole $B$, these values are weaker than the above. Also the percentages are far smaller if we compare the core values with the surface values. Setting up the ratios, we obtain,

$$
(0.0832 / 7.30,0.0783 / 7.16,0.055 / 6.36)=(1.14 \%, 1.09 \%, 0.865 \%)
$$

These values seem reasonable, given that the core radius is $1 \%$ of the surface radius. We are not sure why black hole, $\mathrm{B}$, has a much greater gravitational force both within, and on the surface, when compared to the other values. The entries have been checked more than once, and we find the same results. This anomaly of the truncated Gaussian must have an explanation, but one which is not obvious.

Finally, we consider the entropy, $S_{r}$. Using our by now familiar formalism, for a TE distribution, we find,

$$
\begin{aligned}
& {\left[S_{0.01 R}^{A}, S_{R}^{A}\right]=\left[1.46 \times 10^{36}, 4.01 \times 10^{36}\right]} \\
& {\left[S_{0.01 R}^{B}, S_{R}^{B}\right]=\left[5.75 \times 10^{39}, 1.65 \times 10^{40}\right]} \\
& {\left[S_{0.01 R}^{C}, S_{R}^{C}\right]=\left[5.29 \times 10^{45}, 1.99 \times 10^{46}\right]}
\end{aligned}
$$

The units are in, Joules/K. We see that the largest, i.e., most massive black holes have the highest entropies. But when compared to the values, as predicted by Bekenstein, these values are very low. Bekenstein calculates the entropy as an intrinsic (surface dependent), versus extrinsic (volume dependent) variable. His formula for entropy is, $S_{\text {Bekenstein }}=(1 / 4) c^{3} k_{B} /(G \hbar)\left(4 \pi R^{2}\right)$. Using this formula, the entropy at the surface would calculate to, $\left(1.50 \times 10^{54}, 1.50 \times 10^{56}, 1.50 \times 10^{66}\right)$, all in Joules/K, for black holes $\mathrm{A}, \mathrm{B}$, and, $\mathrm{C}$, respectively. When compare to the surface values in Equations, (5-17), we notice that our values are exceedingly low. As mentioned, our values would seem to indicate that black holes are much more highly ordered states, than what is commonly thought.

It is also noteworthy to mention that the core values for entropy, in Equations, (5-17), are a high percentage of the surface values. This seems to be a pattern for the exponential distribution. If we calculate the percentages, we find,

$$
(1.46 / 4.01,5.75 / 16.5,5.29 / 19.9)=(36 \%, 35 \%, 27 \%)
$$

Within the core we already have a substantial proportion of the total black 
hole entropy.

For comparison purposes, we also give the corresponding values for a truncated Gaussian distribution. Referring to the tables in reference [2], we obtain

$$
\begin{aligned}
& {\left[S_{0.01 R}^{A}, S_{R}^{A}\right]=\left[4.08 \times 10^{35}, 1.63 \times 10^{37}\right]} \\
& {\left[S_{0.01 R}^{B}, S_{R}^{B}\right]=\left[1.60 \times 10^{39}, 6.59 \times 10^{40}\right](\mathrm{TG})} \\
& {\left[S_{0.01 R}^{C}, S_{R}^{C}\right]=\left[1.45 \times 10^{45}, 7.24 \times 10^{46}\right]}
\end{aligned}
$$

These values are comparable to the above, as indicated by Equations, (5-17). And they are also very much less than the Bekenstein entropy values calculated above. In terms of percentages, the core values here are very much less than the surface values. In fact, we find using the numbers in Equations, (5-19), that

$$
(0.0408 / 1.63,0.160 / 6.59,0.145 / 7.24)=(2.5 \%, 2.4 \%, 2.0 \%)(\mathrm{TG})(5-20)
$$

When compared to the corresponding percentages in Equations, (5-18), we notice a large difference. For a truncated Gaussian, the entropy is not concentrated disproportionately at the center.

\section{Conclusion}

Summarizing, by studying various pdf s, we have a rich structure by which to analyze and explore various scenarios for the internal structure of a black hole. If we accept this model of a 4-D spatial ball for a black hole filled with black body radiation (and potentially other forms of radiation), we can investigate various unique characteristics within the black hole. These characteristics within the black hole will lead to specific surface conditions which can be measured from our 3-D perspective. We are thinking of surface forces, surface entropy, surface acceleration, etc., attributes, which will define which pdf is best suited for modeling the interior. In this way valuable insights can be gained, and perhaps, finally, a complete picture as to what is happening within the black hole, and with space itself. This concludes our discussion.

\section{Acknowledgements}

The author would like to thank Gonzaga University, and the physics department, in particular, for an enjoyable and productive collaboration. I would also thank, in particular, my students for their interest, hard work, positive attitude, and spirit. They were second to none.

\section{Conflicts of Interest}

The author declares no conflicts of interest regarding the publication of this paper.

\section{References}

[1] Pilot, C. (2019) Are Black Holes 4-D Spatial Balls Filled with Black Body Radiation? Generalization of the Stefan-Boltzmann Law and Young-Laplace Relation for Spa- 
tial Radiative Transfers. Journal of High Energy Physics, Gravitation and Cosmology, 5, 638-682. https://doi.org/10.4236/jhepgc.2019.53036

[2] Pilot, C. (2019) On the Internal Structure of a Black Hole Utilizing a 4-D Spatial Black Body Radiation Model. Journal of High Energy Physics, Gravitation and Cosmology, 5, 719-772. https://doi.org/10.4236/jhepgc.2019.53039

[3] Johnson, N.L., Kotz, S. and Balakrishnan, N. (1994) Continuous Univariate Distributions. Volume 1, Wiley, Hoboken.

[4] Barr, D.R. and Sherrill, E.T. (1999) Mean and Variance of Truncated Normal Distributions. The American Statistician, 53, 357-361.

https://doi.org/10.1080/00031305.1999.10474490

[5] Burkardt, J. (2018) The Truncated Normal Distribution. Department of Scientific Computing Website, Florida State University, Tallahassee.

[6] Instituto Superior Técnico (2002) Lecture 4: Selection. 1.

[7] Johnson, N.L., Kotz, S. and Balakrishnan, N. (1995) Chapter 21: Beta Distributions. In: Continuous Univariate Distributions, Vol. 2, 2nd Edition, Wiley, Hoboken.

[8] Forge Project; R-Forge Distributions Core Team University Year 2009-2010; Handbook on Probability Distributions.

https://r-forge.r-project.org/scm/viewvc.php/* checkout*/pkg/inst/doc/probdistr-ma in.pdf? revision $=24 \&$ root $=$ distributions $\&$ pathrev $=24$

[9] Another Useful Reference: Handbook of Probability Distribution Functions. http://www.stat.rice.edu/ dobelman/textfiles/DistributionsHandbook.pdf

[10] Carlip, S. (2014) Black Hole Thermodynamics. International Journal of Modern Physics D, 23, Article ID: 1430023. https://doi.org/10.1142/S0218271814300237

[11] Santillan, M., Ares de Parga, G. and Argulo-Brown, F. (1998) Blackbody Radiation and the Maximum Entropy Production Regime. European Journal of Physics, 19, 9. https://www.researchgate.net/publication/215571874 https://doi.org/10.1088/0143-0807/19/4/008

[12] Landsberg, P.T. and De Vos, A. (1989) The Stefan Boltzmann Constant in an N-Dimensional Space. Journal of Physics A: Mathematics and General, 22, 10731084. https://doi.org/10.1088/0305-4470/22/8/021

[13] Menon, V.J. and Agrawal, D.C. (1998) Comment on "The Stefan-Boltzmann Constant in N-Dimensional Space". Journal of Physics A: Mathematics and General, 31, 1109-1110. https://doi.org/10.1088/0305-4470/31/3/021

[14] Caruso, T. and de Castro, A. (2005) The Blackbody Radiation in D-Dimensional Universes.

[15] Bekenstein, J.D. (1973) Black Holes and Entropy. Physical Review D, 7, 2333-2346. https://doi.org/10.1103/PhysRevD.7.2333

[16] Bekenstein, J.D. (1974) The Quantum Mass Spectrum of the Kerr Black Hole. Lettere al Nuovo Cimento, 11, 467. https://doi.org/10.1007/BF02762768

[17] Bekenstein, J.D. (2001) The Limit of Information. Studies in History and Philosophy of Modern Physics, 32, 511-524. 